\title{
STUDIES OF DIFFERENCES FROM THE POINT OF VIEW OF NEVANLINNA THEORY
}

\author{
ZHENG JIANHUA AND RISTO KORHONEN
}

\begin{abstract}
This paper consists of three parts. First, we give so far the best condition under which the shift invariance of the counting function, and of the characteristic of a subharmonic function, holds. Second, a difference analogue of logarithmic derivative of a $\delta$-subharmonic function is established allowing the case of hyper-order equal to one and minimal hyper-type, which improves the condition of the hyper-order less than one. Finally, we make a careful discussion of a well-known difference equation and give out the possible forms of the equation under a growth condition for the solutions.
\end{abstract}

\section{Introduction and Main Results}

One of the main purposes of Nevanlinna theory in the study of difference equations is to single out those equations which are assumed to have meromorphic solutions of finite order and then to make a further careful analysis of the singledout difference equations by local singularity analysis and other methods. From the point of view of discrete Painlevé equations, much interest has been attracted by the following algebraic difference equations

$$
\begin{gathered}
\bar{w}=R(z, w), \\
\bar{w}+\underline{w}=R(z, w), \\
\bar{w} \underline{w}=R(z, w), \\
(\bar{w}+w)(w+\underline{w})=R(z, w),
\end{gathered}
$$

where, henceforth, $\bar{w}=w(z+1), \underline{w}=w(z-1)$ and $R(z, w)$ is a rational function in $z$ and $w$. The eye-catching reason is that Nevanlinna theory has proved to be powerful in the study of these kinds of difference equations, although they have widely been considered by other methods. The basic method, when implementing Nevanlinna theory to study the equations above, consists of two steps: The first is that in view of the Valiron-Mohon'ko Theorem ([21] [18]), an estimate of the degree of $R(z, w)$ in $w$ is obtained, in fact, ruling out the higher degree equations. These type of results may be called difference Malmquist type theorems. As we know, $\operatorname{deg}_{w}(R) \leq 1$ for (1.1) $([23]) ; \operatorname{deg}_{w}(R) \leq 2$ for (11.2) and (1.3) $([1]) ; \operatorname{deg}_{w}(R) \leq 4$ for (1.4) ([8]) if those difference equations are assumed to have a transcendental meromorphic solution of finite order. The second step is that the equations are classified into several

2000 Mathematics Subject Classification: Primary 39A10, Secondary 30D35, 39A12 The first author is partially supported by the grant (No. 11571193) of NSF of China. The second author is supported in part by the Academy of Finland grant 286877. 
special types by analyzing the behavior near poles of a meromorphic solution. The basic idea here is related to singularity confinement, but the analysis goes deeper. This analysis reduces the above equations into linear equations, or the Riccati equation with degree one, or to one of the difference Painlevé equations (see, e.g., [9]). To determine high pole density of meromorphic solutions is an application of a difference Clunie type theorem ([5], [17] and [15]).

A difference rational function is a fraction of two irreducible difference polynomials and its total degree is the maximal one of degrees of the numerator and the denominator. Let us consider the following difference polynomial equation

$$
U(z, \vec{w}) P(z, \vec{w})=Q(z, \vec{w})
$$

where $U, P$ and $Q$ are three difference polynomials in $\vec{w}(z)=\left(w(z), w\left(z+c_{1}\right), \ldots, w(z+\right.$ $\left.c_{n}\right)$ ) for non-zero complex numbers $c_{j}$ and $U P$ does not have common factors with $Q$ with respect to $\vec{w}$. We call the equation (1.5) Clunie type equation after J. Clunie who is the first one to research such a differential polynomial equations ([5]).

In view of the method above to study this topic, we first establish a difference Malmquist type theorem, that is to say, find a relationship among the degrees of three difference polynomials $U, P$ and $Q$. According to the well-known methods in terms of Nevanlinna theory, we should first establish a difference version of the Valiron-Mohon'ko Theorem, in the other words, we are asked to extend the ValironMohon'ko Theorem to the case of difference polynomials dealing with the shifts of the function in question. Therefore, we are forced to consider when

$$
T(r, w(z+c)) \sim T(r, w(z))
$$

as $r \rightarrow \infty$ on at least a sequence of positive numbers tending to $+\infty$ for all finite complex numbers $c$ where $T(r, w)$ denotes the Nevanlinna characteristic of $w$. (For basic definitions of Nevanlinna theory, we refer to [13, 26].) It is easily seen that (1.6) is essentially fundamental in the sense that existence of a transcendental meromorphic solution with (1.6) of difference equations (1.1) - (1.4) derives easily the above-mentioned estimates of the degrees in $w$ of the rational function $R(z, w)$. Generally, we can establish Theorem 4.1 (see Section 4 below) which shows the significance of (1.6).

Unfortunately, (1.6) does not generally hold. An explicit example is $w(z)=e^{e^{z}}$ which is such that $T(r, w(z+1))=m(r, w(z+1))=e m(r, w(z))=e T(r, w)$ for all $r>0$. From the point of view of difference equations, it is easy to find such an example so that (1.6) does not hold. It is proved in 20 that for any non-constant polynomial $P(w)$, the difference equation $w(z+1)=P(w(z))$ has a non-trivial entire solution. If, in addition, the degree of $P$ satisfies $\operatorname{deg}(P)>1$, then none of the sequences of positive numbers tending to $\infty$ is such that (1.6) holds for the entire solution $w$. A generalization is made in 23 with the $P$ replaced by a rational function $R$, according to which the difference equation $w(z+1)=R(w(z))$ always has a non-trivial meromorphic solution $w$ and for such a solution $w$, we have $T(r, \bar{w})=\operatorname{deg}_{w}(R) T(r, w)+O(1)$, and hence

$$
T(r, w) \geq K D^{r}, \quad \forall r>0,
$$


where $D$ is any positive number less than the $\operatorname{degree~} \operatorname{deg}_{w}(R)$ of $R$ and $K$ is a positive constant depending on $D$. Conversely, these facts give us a heads up that (1.6) may be true if $\log T(r, w)=o(r)(r \rightarrow \infty)$.

Studying the possibility of (1.6) we may ascend to works of many great mathematicians of the last century, such as Valiron, Dugúe, Hayman, Goldberg and Ostrovskii, only mentioning some of them here. The reason to study this topic is very natural, as Goldberg and Ostrovskii pointed out in their monograph [7]. All of the functions with the form $f(q z+c)$ map the complex plane onto the same Riemann surface. It thus makes sense to study the invariance of quantities characterizing the asymptotic behavior of $w(z)$ under the linear transformation $q z+c$ which can be decomposed into $q z$ and $z+c$. The change produced by $q z$ is clear, so the attention is put on the shifting by $c$. From the point of view of Ahlfors theory of covering surfaces, the asymptotic behavior of $w(z)$ is characterized with the help of the covering area under image of $w$ from a disk, and the change of a disk by a translating can be handled. In view of this observation, we can establish

$$
T\left(r, w_{c}\right) \leq T(r+|c|, w)+\frac{(2+|c|) \log (1+|c|)}{\log (r+|c|)} T(r+|c|, w)+O(1),
$$

here and henceforth, $w_{c}$ denotes $w(z+c)$. The details will be provided in Section 2 below. Therefore, by noting that $w=\left(w_{c}\right)_{-c}$, (1.6) is naturally transferred into the discussion of possibility of the equivalent relation

$$
T(r+h, w) \sim T(r, w),
$$

as $r \rightarrow \infty$ on at least a sequence of positive numbers tending to $+\infty$ for any positive number $h$. However, the number $h$ can be chosen as 1 without loss of generality. Following the discussion of invariance of Nevanlinna deficiency under a change of the origin given by Goldberg and Ostrovskii ([7]), we can establish the following result.

Theorem 1.1. Let $w$ be a transcendental meromorphic function with

$$
\liminf _{r \rightarrow \infty} \frac{\log T(r, w)}{r}=0 .
$$

Then (1.6) and (1.7) for $T(r, w)$ and $N(r, w)$ hold as $r \notin E \rightarrow \infty$, where $E$ is a subset of $[1,+\infty)$ with the zero lower density. If liminf in (1.8) is replaced by limsup, then $E$ can be taken as a set of zero upper density.

An upper density of a set $E$ in $[1,+\infty)$ is defined as

$$
\overline{\operatorname{dens}} E=\limsup _{r \rightarrow \infty} \frac{1}{r} \int_{E[1, r]} \mathrm{d} t,
$$

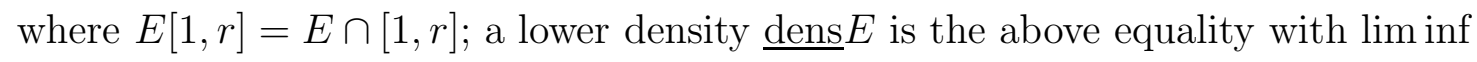
replacing limsup.

In fact, (1.8) in Theorem 1.1 is true for all non-decreasing functions $T(r)$ that are convex with respect to $\log r$. Above $N(r, w)$ denotes the integrated counting function of $w$. If $N(r, w) \neq 0$, that is to say, $w$ has at least a pole, then $N(r, w)$ is a non-decreasing convex function with respect to $\log r$. And $N(r, w)$ satisfies (1.8) 
if (1.8) holds for $T(r, w)$. Therefore, in this case, Theorem 1.1 applies to $N(r, w)$. By noting that $N(r, w) \leq T(r, w)$, we have

$$
T\left(r, w_{c}\right)=(1+\varepsilon(r)) T(r, w), N\left(r, w_{c}\right)=\left(1+\varepsilon^{\prime}(r)\right) N(r, w),
$$

where $\varepsilon(r)\left(\right.$ resp. $\left.\varepsilon^{\prime}(r)\right) \rightarrow 0(r \notin E \rightarrow \infty)$ and it is defined in the proof of Theorem 1.1 and expressed in terms of $T(r, w)$ (resp. $N(r, w))$. Then we have

$$
m\left(r, w_{c}\right)=(1+\varepsilon(r)) m(r, w)+\left(\varepsilon(r)-\varepsilon^{\prime}(r)\right) N(r, w) .
$$

This produces that

$$
m\left(r, w_{c}\right)-m(r, w)=o(T(r, w)) .
$$

From the Clunie type theorems obtained in the literature, we know that $m(r, w)$ is often small with respect to $T(r, w)$, roughly speaking, it may be lost sight of. When this happens, $w$ has high pole density in the sense of Nevanlinna theory, which is a starting point of making a further classification of some difference equations by singularity confinement type arguments, but we cannot confirm $m\left(r, w_{c}\right) \sim$ $m(r, w)(r \notin E \rightarrow \infty)$, basically. In some cases (see the discussion in Section 4), the equivalent relation $m\left(r, w_{c}\right) \sim m(r, w)(r \notin E \rightarrow \infty)$ is often useful.

However, usually the most useful estimate is concerned with the proximity function of the ratio of $w_{c}$ and $w$, known as the lemma of the logarithmic differences, which occupies the same important position as the lemma of logarithmic derivative does in Nevanlinna theory. For example, in establishing the Clunie type theorems and the Nevanlinna second main theorem for differences ([12] and [16]), it is a crucial part. Such lemmas for meromorphic functions of finite order were established independently by Halburd and the second author in [11 and Chiang and Feng in [3].

We cannot obtain a valuable estimate of $m\left(r, \frac{w_{c}}{w}\right)$ from (1.9), although we know that

and

$$
m\left(r, w_{c}\right) \leq m(r, w)+m\left(r, \frac{w_{c}}{w}\right)
$$

$$
m(r, w) \leq m\left(r, w_{c}\right)+m\left(r, \frac{w}{w_{c}}\right)
$$

Indeed, conversely, an estimate of $m\left(r, \frac{w_{c}}{w}\right)$ will produce an estimate of $\mid m\left(r, w_{c}\right)$ $m(r, w) \mid$.

In [12, Halburd, the second author and Tohge established a version of the lemma of the logarithmic differences covering the result of finite order and dealing with the case of infinite order.

Theorem A. Let $w(\not \equiv 0)$ be a meromorphic function with the hyper-order $\zeta=$ $\zeta(w)<1$. Then for each $\varepsilon>0$, we have

$$
m\left(r, \frac{w_{c}}{w}\right)=o\left(\frac{T(r, w)}{r^{1-\zeta-\varepsilon}}\right), r \notin E .
$$

If the order $\rho(w)<+\infty$, then

$$
m\left(r, \frac{w_{c}}{w}\right) \leq \frac{(\log r)^{3+\varepsilon}}{r} T(r, w), r \notin E .
$$


Here $E \subset[1,+\infty)$ is a set of finite logarithmic measure, i.e., $\int_{E} \frac{\mathrm{d} t}{t}<\infty$.

The hyper-order $\zeta(w)$ of $w$ is defined by

$$
\zeta(w):=\limsup _{r \rightarrow \infty} \frac{\log \log T(r, w)}{\log r}
$$

and the lower hyper-order by replacing limsup with lim inf. Obviously, (1.10) is invalid when $\zeta=1$. In this paper, we consider the case when the growth does not exceed the lower hyper-order 1 and minimal lower hyper-type, i.e., (1.8). We can establish the following result, which is a special case of Theorem 3.2 below.

Theorem 1.2. Let $w(\not \equiv 0)$ be a meromorphic function. Assume that (1.8) holds. Then for a complex number $c$ and a real number $\delta$ with $0<\delta<1 / 2$, we have

$$
m\left(r, \frac{w_{c}}{w}\right) \leq 436 e(1+|c|)\left(\min _{1 \leq t \leq r} \frac{\log T(t, w)}{t}\right)^{\delta} T(r, w), r \notin E_{\delta},
$$

where $E_{\delta}$ is a subset of $[1,+\infty)$ with $\underline{\text { dens }} E_{\delta}=0$ and independent of $c$. If (1.8) holds for limsup, then $\overline{\operatorname{dens}} E_{\delta}=0$.

Clearly, (1.8) implies that

$$
\min _{1 \leq t \leq r} \frac{\log T(t, w)}{t} \rightarrow 0(r \rightarrow \infty)
$$

Therefore, from (1.11) it follows that for any $c$ with $0 \leq|c| \leq o\left(\max _{1 \leq t \leq r} \frac{t}{\log T(t, w)}\right)^{\delta}$, we have

$$
m\left(r, \frac{w_{c}}{w}\right)=o(T(r, w)), r \notin E_{\delta} \rightarrow \infty .
$$

Obviously, we can require $o\left(\max _{1 \leq t \leq r} \frac{t}{\log T(t, w)}\right)^{\delta} \rightarrow \infty(r \rightarrow \infty)$.

The exception set $E_{\delta}$ has zero lower density, however, the situation we often meet, for example, in the second main theorem of Nevanlinna, is that the exception set is of finite logarithmic measure. We will pay a price on the growth of meromorphic functions if we ask the exception set of finite logarithmic measure.

Theorem 1.3. Let $w(\not \equiv 0)$ be a meromorphic function. Then for a complex number $c$, a real number $\delta$ with $0<\delta<1 / 2$ and $\varepsilon>0$, we have

$$
m\left(r, \frac{w_{c}}{w}\right) \leq 436 e(1+|c|)\left(\frac{(\log \log T(r, w))^{1+\varepsilon} \log T(r, w)}{r}\right)^{\delta} T(r, w), r \notin E,
$$

where $E$ is a subset of $[1,+\infty)$ with finite logarithmic measure.

Let us make a remark. If

$$
\limsup _{r \rightarrow \infty} \frac{(\log r)^{1+\varepsilon} \log T(r, w)}{r}=0,
$$

then

$$
\frac{(\log \log T(r, w))^{1+\varepsilon} \log T(r, w)}{r} \rightarrow 0(r \rightarrow \infty)
$$


The $w$ satisfying (1.12) may have the hyper-order $\zeta(w)=1$. The following is a consequence of Theorem 1.3 .

Theorem 1.4. Let $w(\not \equiv 0)$ be a meromorphic function. Assume that (1.12) holds for some $\varepsilon>0$. Then for $0<\delta<1 / 2$ and for any complex number $c$ with

$$
0 \leq|c| \leq o\left(\frac{r}{(\log r)^{1+\varepsilon} \log T(r, w)}\right)^{\delta},
$$

we have

$$
m\left(r, \frac{w_{c}}{w}\right)=o(T(r, w)), r \notin E .
$$

Here $E$ is a subset of $[1,+\infty)$ with finite logarithmic measure.

We can take $o\left(\frac{r}{(\log r)^{1+\varepsilon} \log T(r, w)}\right) \rightarrow \infty(r \rightarrow \infty)$ under (1.12). Then $c$ is allowed to tend to $\infty$ as long as $r$ goes to $\infty$. If $c$ is a fixed complex number, then we still have (1.13) even if (1.12) is replaced by

$$
\lim _{\varepsilon \rightarrow 0^{+}} \limsup _{r \rightarrow \infty} \frac{(\log r)^{1+\varepsilon} \log T(r, w)}{r}=0 .
$$

In fact, under this condition, set for $\varepsilon>0$

$$
S(\varepsilon)=\limsup _{r \rightarrow \infty} \frac{(\log \log T(r, w))^{1+\varepsilon} \log T(r, w)}{r} .
$$

Then $\lim _{\varepsilon \rightarrow 0^{+}} S(\varepsilon)=0$. Assume without any loss of generality that $S(\varepsilon)>0, \forall \varepsilon>0$. There exists an $r_{\varepsilon}>0$ such that for $r \geq r_{\varepsilon}$, we have

$$
\frac{(\log \log T(r, w))^{1+\varepsilon} \log T(r, w)}{r}<2 S(\varepsilon) .
$$

In view of Theorem 1.3 , there exists a subset $E_{\varepsilon}$ of $[1,+\infty)$ with finite logarithmic measure such that

$$
m\left(r, \frac{w_{c}}{w}\right) \leq 436 e(1+|c|)(2 S(\varepsilon))^{\delta} T(r, w), r \notin E_{\varepsilon}, r \geq r_{\varepsilon} .
$$

Take a sequence $\left\{\varepsilon_{n}\right\}$ with $0<\varepsilon_{n+1}<\varepsilon_{n} \rightarrow 0(n \rightarrow \infty)$ and a sequence $\left\{r_{n}\right\}$ of positive numbers with $r_{n} \geq r_{\varepsilon_{n}}$ and $r_{n+1} \geq r_{n} \rightarrow \infty(n \rightarrow \infty)$ such that

$$
\int_{F_{n}} \frac{\mathrm{d} t}{t}<\frac{1}{2^{n}}, F_{n}=E_{\varepsilon_{n}} \cap\left[r_{n}, r_{n+1}\right) .
$$

Set $E=\left[1, r_{1}\right) \cup\left(\cup_{n=1}^{\infty} F_{n}\right)$. Then $E$ has finite logarithmic measure. Then for $r \notin E$ with $r_{n} \leq r<r_{n+1}$ we have

$$
m\left(r, \frac{w_{c}}{w}\right) \leq 436 e(1+|c|)\left(2 S\left(\varepsilon_{n}\right)\right)^{\delta} T(r, w)=o(T(r, w)),
$$

by noting that $S\left(\varepsilon_{n}\right) \rightarrow 0(r \rightarrow \infty)$.

In this paper, we will consider the differences of $\delta$-subharmonic functions. Thus the results we obtain will apply to the meromorphic functions, the holomorphic curves and the algebroid functions. That is to say, the results stated above are special cases of the results we obtain in the sequel. Therefore, the second main 
theorems of holomorphic curves for the difference operator ([12] and [16]) will be available for the growth not exceeding the (lower) hyper-order one and minimal (lower) hyper-type. And we can also establish the difference analogue of the second main theorem for algebroid functions with the hyper-order at most one and having minimal hyper-type.

\section{SHIFT INVARIANCE OF $N_{s}$ AND $T_{s}$}

A meromorphic function can be considered as a holomorphic curve of dimension one and an algebroid function can be also expressed by the coefficients of its corresponding algebraic equation as a holomorphic curve. However, we can observe holomorphic curves from the potential point of view. Therefore, this leads us to consider the difference of subharmonic functions.

Let $u$ be a $\delta$-subharmonic function on $\mathbb{C}$. Let $D$ be a domain on $\mathbb{C}$ surrounded by finitely many piecewise analytic curves. Then for any point $z \in D$, we have

$$
u(z)=\frac{1}{2 \pi} \int_{\partial D} u(\zeta) \frac{\partial G_{D}(z, \zeta)}{\partial \mathbf{n}} \mathrm{d} s-\frac{1}{2 \pi} \int_{D} G_{D}(z, w) \Delta u(w),
$$

where $G_{D}(z, w)$ is the Green function for $D$ with singularity at $z, \mathbf{n}$ is the inner normal of $\partial D$ with respect to $D$ and $\Delta$ is the Laplacian. (Please see [19] for the basic theory of subharmonic functions). Define

$$
\begin{aligned}
& m_{s}(D, z, u):=\frac{1}{2 \pi} \int_{\partial D} u^{+}(\zeta) \frac{\partial G_{D}(z, \zeta)}{\partial \mathbf{n}} \mathrm{d} s \\
& N_{s}(D, z, u):=\frac{1}{2 \pi} \int_{D} G_{D}(z, w)(\Delta u)^{-}(w),
\end{aligned}
$$

where $u^{+}=\max \{0, u\}$ and $(\Delta u)^{-}$is the negative variation of $\Delta u$, and

$$
T_{s}(D, z, u):=m_{s}(D, z, u)+N_{s}(D, z, u) .
$$

$T_{s}$ is known as the Nevanlinna characteristic, where the subscript $s$ stands for "subharmonic" function, which is used to distinguish it from that of a characteristic of a meromorphic function. That is to say, the functions $m_{s}, N_{s}$ and $T_{s}$ are defined for $\delta$-subharmonic functions. The Poisson-Jensen formula (2.1) implies that

$$
u(z)=T_{s}(D, z, u)-T_{s}(D, z,-u) .
$$

Now let $D$ be a disk $B(0, r)=\{z:|z|<r\}$. We write $m_{s}(r, u), N_{s}(r, u)$ and $T_{s}(r, u)$ for $m_{s}(D, 0, u), N_{s}(D, 0, u)$ and $T_{s}(D, 0, u)$, respectively. As we know, $N_{s}(r, u)$ is non-decreasing and logarithmically convex in $r$, i.e., convex in $\log r$ and has the form

$$
N_{s}(r, u)=\int_{0}^{r} \frac{n_{s}(t, u)-n_{s}(0, u)}{t} \mathrm{~d} t+n_{s}(0, u) \log r
$$

where $n_{s}(t, u)=\frac{1}{2 \pi}(\Delta u)^{-}(B(0, t))$.

Assume that $u$ is subharmonic. Then $(\Delta u)^{-}=0$ and so applying (2.1) to the subharmonic function $u^{+}$yields

$$
T_{s}(r, u)=m_{s}(r, u)=N_{s}\left(r,-u^{+}\right)+u^{+}(0) .
$$


By noting that $-u^{+}$is $\delta$-subharmonic, it follows that $T_{s}(r, u)$ is non-decreasing and logarithmically convex in $r$. For a subharmonic function $u$, we often use the Cartan characteristic which is defined by

$$
\mathcal{T}_{s}(r, u):=\frac{1}{2 \pi} \int_{0}^{2 \pi} u\left(r e^{i \theta}\right) \mathrm{d} \theta=N_{s}(r,-u)+u(0) .
$$

Hence, $\mathcal{T}_{s}(r, u)$ is non-decreasing and logarithmically convex in $r$. It is enough to focus on the discussion of the shift invariance of $N_{s}$ of $u$ by $c$ for our purposes in this section.

Let $\mathcal{P}^{n}(\mathbb{C})$ be the $n$-dimensional complex projective space, that is, $\mathcal{P}^{n}(\mathbb{C})=$ $\mathbb{C}^{n+1} \backslash\{0\} / \sim$, where $\sim$ is the equivalence relation defined so that $\left(a_{0}, a_{1}, \ldots, a_{n}\right) \sim$ $\left(b_{0}, b_{1}, \ldots, b_{n}\right)$ if and only if $\left(a_{0}, a_{1}, \ldots, a_{n}\right)=\lambda\left(b_{0}, b_{1}, \ldots, b_{n}\right)$ for some $\lambda \in \mathbb{C} \backslash\{0\}$. We write the equivalence class of $\left(a_{0}, a_{1}, \ldots, a_{n}\right)$ as $\left[a_{0}: a_{1}: \ldots: a_{n}\right]$.

A map $f: \mathbb{C} \rightarrow \mathcal{P}^{n}(\mathbb{C})$ is called a holomorphic curve, if we can write $f=\left[f_{0}\right.$ : $\left.f_{1}: \ldots: f_{n}\right]$ where every $f_{j}$ is an entire function, at least one of $f_{j}$ is non-constant and they have no common zeros on $\mathbb{C}$, and $\mathbf{f}=\left(f_{0}, f_{1}, \ldots, f_{n}\right)$ is called a reduced representation of $f$.

Let $f$ be a holomorphic curve on the complex plane $\mathbb{C}$ with a reduced representation $\mathbf{f}=\left(f_{0}, f_{1}, \ldots, f_{n}\right)$. Set

$$
v_{f}(z)=\bigvee_{j=0}^{n} \log \left|f_{j}(z)\right| .
$$

Then $v_{f}$ is subharmonic on $\mathbb{C}$. The Cartan characteristic of $f$ is defined by

$$
\mathcal{T}(r, f)=\mathcal{T}_{s}\left(r, v_{f}\right)-v_{f}(0)=\frac{1}{2 \pi} \int_{0}^{2 \pi} v_{f}\left(r e^{i \theta}\right) \mathrm{d} \theta-v_{f}(0) .
$$

Then $\mathcal{T}(r, f)$ is a positive logarithmically convex increasing real-valued function in $r$. If at least one of $f_{j}(j=0,1,2, \ldots, n)$ is transcendental, then $\mathcal{T}(r, f) / \log r \rightarrow$ $\infty(r \rightarrow \infty)$. We can write the characteristic of an algebroid function as a special holomorphic curve, by this way. We can consider a meromorphic function $f$ as a holomorphic curve and have its characteristic $\mathcal{T}(r, f)$, which is known as the Ahlfors-Shimizu characteristic. The Nevanlinna characteristic $T(r, f)$ of a meromorphic function $f$ is

$$
T(r, f)=T_{s}(r, \log |f|),
$$

by noting that $\log |f|$ is a $\delta$-subharmonic function. The following inequality is well known (see, for example, [13] and [26])

$$
|T(r, f)-\mathcal{T}(r, f)| \leq \log 2+\log ^{+}|f(0)| .
$$

In a word, all of the following results are valid with $\mathcal{T}_{s}(r, u)$ for subharmonic functions $u$, with $\mathcal{T}(r, f)$ for holomorphic curves $f$ including the algebroid functions, and with $T(r, f)$ for meromorphic functions $f$.

Theorem 2.1. Let $u$ be a $\delta$-subharmonic function on $\mathbb{C}$. Then we have

$$
N_{s}\left(r, u_{c}\right) \sim N_{s}(r, u) \text {, as } r \notin E \rightarrow \infty,
$$

where $E$ is a subset of $[1,+\infty)$ such that 
(1) if

$$
\liminf _{r \rightarrow \infty} \frac{\log N_{s}(r, u)}{r}=0
$$

then $\underline{\text { dens }} E=0$;

(2) if (2.2) holds for limsup instead of liminf, then $\overline{\operatorname{dens}} E=0$;

(3) if

$$
\limsup _{r \rightarrow \infty} \frac{(\log r)^{1+\varepsilon} \log N_{s}(r, u)}{r}=0,
$$

then E has finite logarithmic measure.

Let us point out that Theorem 2.1 holds for $T_{s}, \mathcal{T}_{s}, T$ and $\mathcal{T}$ instead of $N_{s}$. Theorem 1.1 follows from Theorem 2.1 .

In order to prove Theorem 2.1, we need a basic lemma some of whose idea essentially comes from [7].

Lemma 2.1. Let $T(r)$ be a non-decreasing positive function in $[1,+\infty)$ and logarithmically convex with $T(r) \rightarrow+\infty(r \rightarrow+\infty)$. Assume that

$$
\liminf _{r \rightarrow \infty} \frac{\log T(r)}{r}=0 \text {. }
$$

Set

$$
\phi(r)=\max _{1 \leq t \leq r}\left\{\frac{t}{\max \{1, \log T(t)\}}\right\} .
$$

Then given a real number $\delta$ with $0<\delta<\frac{1}{2}$, we have

$$
T\left(r+\phi^{\delta}(r)\right) \leq T(r)+4 \phi(r)^{\delta-\frac{1}{2}} T(r), r \notin E_{\delta},
$$

where $E_{\delta}$ is a subset of $[1,+\infty)$ with the zero lower density. And $E_{\delta}$ has the zero density if (2.4) holds for limsup.

Proof. Since $T(r)$ is logarithmically convex, it follows from the basic inequality of a convex function that for any $t, r \in[1,+\infty)$, we have

$$
T(r) \geq T(t)+\frac{\mathrm{d} T(t)}{\mathrm{d} \log t}(\log r-\log t)
$$

so that

$$
T(t) \leq T(r)+\frac{\mathrm{d} T(t)}{\mathrm{d} t} t \log \frac{t}{r}
$$

Take $t=r+\phi^{\delta}(r)$. By noting that $\phi(r) \leq r$, we have

$$
\begin{aligned}
T\left(r+\phi^{\delta}(r)\right) & \leq T(r)+\frac{T^{\prime}\left(r+\phi^{\delta}(r)\right)}{T\left(r+\phi^{\delta}(r)\right)}\left(r+\phi^{\delta}(r)\right) \log \left(1+\frac{\phi^{\delta}(r)}{r}\right) T\left(r+\phi^{\delta}(r)\right) \\
(2.5) & \leq T(r)+\frac{T^{\prime}\left(r+\phi^{\delta}(r)\right)}{T\left(r+\phi^{\delta}(r)\right)} 2 \phi^{\delta}(r) T\left(r+\phi^{\delta}(r)\right) .
\end{aligned}
$$

Defining

$$
\hat{\tau}(r)=\sqrt{\frac{\log ^{+} T(r)}{r}}, r \in[1,+\infty),
$$


it follows that $\hat{\tau}$ is continuous in $[1,+\infty)$. From (2.4) we can find a sequence of positive numbers $\left\{r_{n}\right\}$ such that $r_{1}=1, r_{n}<r_{n+1} \rightarrow \infty(n \rightarrow \infty)$ and $\hat{\tau}\left(r_{n}\right)=$ $\min _{1 \leq t \leq r_{n}} \hat{\tau}(t)$. It is obvious that $0<\hat{\tau}\left(r_{n+1}\right) \leq \hat{\tau}\left(r_{n}\right) \rightarrow 0(n \rightarrow \infty)$. Define $\tau(r)=$ $\hat{\tau}\left(r_{n}\right), r_{n-1}<r \leq r_{n}$. It is easy to see that $\tau(r)$ is non-increasing in $[1,+\infty), \tau(r)=$ $\phi(r)^{-1 / 2}$ for $r=r_{n}$ and $\tau(r) \leq \phi(r)^{-1 / 2} \rightarrow 0(r \rightarrow \infty)$, as $\phi(r) \rightarrow \infty(r \rightarrow \infty)$ from (2.4).

Set

$$
F=\left\{r \in[1,+\infty): \frac{T^{\prime}(r)}{T(r)} \geq \tau(r)\right\} .
$$

There is an $r_{0}>1$ such that $T\left(r_{0}\right) \geq 1$. Then

$$
\begin{aligned}
\log T(r) & \geq \int_{r_{0}}^{r}(\log T(t))_{t}^{\prime} \mathrm{d} t \\
& =\int_{r_{0}}^{r} \frac{T^{\prime}(t)}{T(t)} \mathrm{d} t \\
& \geq \int_{F\left[r_{0}, r\right]} \frac{T^{\prime}(t)}{T(t)} \mathrm{d} t \\
& \geq \int_{F\left[r_{0}, r\right]} \tau(t) \mathrm{d} t \\
& \geq \tau(r) \int_{F\left[r_{0}, r\right]} \mathrm{d} t
\end{aligned}
$$

where $F\left[r_{0}, r\right]=F \cap\left[r_{0}, r\right]$ so that, when $r=r_{n}$, we have

$$
\begin{aligned}
\frac{1}{r} \int_{F[1, r]} \mathrm{d} t & =\frac{1}{r} \int_{F\left[1, r_{0}\right]} \mathrm{d} t+\frac{1}{r} \int_{F\left[r_{0}, r\right]} \mathrm{d} t \\
& \leq \frac{1}{r} \int_{1}^{r_{0}} \mathrm{~d} t+\frac{\hat{\tau}^{2}(r)}{\tau(r)} \\
& =\frac{r_{0}-1}{r}+\tau(r) \rightarrow 0(r \rightarrow \infty) .
\end{aligned}
$$

This yields that the lower density of $F$ satisfies $\underline{\operatorname{dens}}(F)=0$. Set $E_{\delta}=\{r$ : $\left.r+\phi^{\delta}(r) \in F\right\}$. Since $r+\phi^{\delta}(r) \sim r(r \rightarrow \infty)$, we have $\underline{\operatorname{dens}}\left(E_{\delta}\right)=0$. There is an $R_{0}>1$ such that $2 \phi^{\delta}(r) \tau(r) \leq 2 \phi^{\delta-1 / 2}(r)<1 / 2$ for $r>R_{0}$. Then for $\forall r \notin E_{\delta} \cup\left[1, R_{0}\right]$, we have

$$
\begin{aligned}
T\left(r+\phi^{\delta}(r)\right) & \leq T(r)+2 \phi^{\delta}(r) \tau\left(r+\phi^{\delta}(r)\right) T\left(r+\phi^{\delta}(r)\right) \\
& \leq T(r)+2 \phi^{\delta}(r) \tau(r) T\left(r+\phi^{\delta}(r)\right) \\
& <T(r)+\frac{1}{2} T\left(r+\phi^{\delta}(r)\right)
\end{aligned}
$$

which reduces to $T\left(r+\phi^{\delta}(r)\right)<2 T(r)$. Thus for $r \notin E_{\delta} \cup\left[1, R_{0}\right]$,

$$
T\left(r+\phi^{\delta}(r)\right) \leq T(r)+4 \phi^{\delta}(r) \tau(r) T(r) \leq T(r)+4 \phi(r)^{\delta-1 / 2} T(r) .
$$


Let us make a remark on Lemma 2.1. As we know, there are a few lemmas on the growth of real functions that were established in Nevanlinna theory, so we wonder if from them we could obtain Lemma 2.1. After checking this, we have not found the possibility. Set

$$
E=\{r \geq 1: T(r+1) \geq C T(r)\}, C>1
$$

In view of Lemma 3.3.1 in [2], we have

$$
\begin{aligned}
\int_{E[e, r]} \frac{\mathrm{d} t}{t} & \leq \frac{1}{T^{-1}(e)}+\frac{1}{\log C} \int_{e}^{T(r)} \frac{\mathrm{d} t}{t T^{-1}(t)} \\
& =\frac{1}{T^{-1}(e)}+\frac{1}{\log C} \int_{T^{-1}(e)}^{r} \frac{T^{\prime}(t)}{T(t) t} \mathrm{~d} t \\
& =\frac{1}{T^{-1}(e)}+\frac{1}{\log C}\left(\frac{\log T(r)}{r}-\frac{1}{T^{-1}(e)}+\int_{T^{-1}(e)}^{r} \frac{\log T(t)}{t^{2}} \mathrm{~d} t\right) .
\end{aligned}
$$

Therefore, if (2.4) holds for limsup, then $E$ has the upper logarithmic density $\overline{\log d e n s}(E)=0$. As we know that $\overline{\log \operatorname{dens}}(E) \leq \overline{\operatorname{dens}}(E)$, from Lemma 3.3.1 in [2] we cannot have $\overline{d e n s}(E)=0$. And from $T(r+1)<C T(r)$ with $C>1$ for $r \notin E$ we cannot directly obtain $T(r+1) \sim T(r)(r \rightarrow \infty)$.

If the growth of $T(r)$ is a little slower, we can obtain that the exceptional set $E_{\delta}$ has finite logarithmic measure, which appears often in the second main theorems of Nevanlinna. The following is Lemma 10.1 of Edrei and Fuchs [6].

Lemma 2.2. Let $\psi$ and $\varphi$ be two positive functions on $\left[r_{0},+\infty\right)$ with $r_{0}>0$. Assume that $\psi$ is non-decreasing while $\varphi$ is non-increasing and that $\psi\left(r_{1}\right)>r_{0}+1$ for some $r_{1}>r_{0}$. Set

$$
E=\left\{r \geq r_{1}: \psi(r+\varphi(\psi(r))) \geq \psi(r)+1\right\} .
$$

Then we have

$$
\int_{E[a, A]} \mathrm{d} t \leq \int_{\psi(a)-1}^{\psi(A)} \varphi(t) \mathrm{d} t
$$

provided that $r_{1} \leq a<A<+\infty$, where $E[a, A]=E \cap[a, A]$.

For a non-decreasing positive function $T(r)$ on $[1,+\infty)$ with $T(r)>e$, define

$$
\psi(r)=\log T\left(e^{r}\right), \varphi(r)=\frac{1}{r(\log r)^{1+\varepsilon}} .
$$

Then

$$
\begin{aligned}
\psi(\log r+\varphi(\psi(\log r))) & =\log T\left(r e^{1 /\left(\log T(r)(\log \log T(r))^{1+\varepsilon}\right)}\right) \\
& \leq \psi(\log r)+1 \\
& =\log e T(r), \quad \log r \notin F_{\varepsilon},
\end{aligned}
$$

where $F_{\varepsilon}$ is a subset of $[0,+\infty)$ such that

$$
\int_{F_{\varepsilon}[1, r]} \mathrm{d} t \leq \int_{1}^{\infty} \varphi(t) \mathrm{d} t<+\infty,
$$


that is to say, $F_{\varepsilon}$ has finite linear measure. Set $E_{\varepsilon}=\left\{r>e: \log r \in F_{\varepsilon}\right\}$. Then $E_{\varepsilon}$ has finite logarithmic measure and

$$
T\left(r e^{1 /\left(\log T(r)(\log \log T(r))^{1+\varepsilon}\right)}\right) \leq e T(r), r \notin E_{\varepsilon} .
$$

Here and henceforth, define

$$
\phi_{T, \varepsilon}(r)=\frac{r}{(\log \log T(r))^{1+\varepsilon} \log T(r)}
$$

for a non-decreasing positive function $T(r)$ with $T(r)>e$ and $\varepsilon>0$. Without confusion in the context, we will write $\phi_{\varepsilon}$ for $\phi_{T, \varepsilon}$. Then (2.6) produces

$$
T\left(r+\phi_{\varepsilon}(r)\right) \leq e T(r), r \notin E_{\varepsilon} .
$$

As an application of (2.7), we establish the following, which is a supplement of Lemma 2.1. We emphasize that these two lemmas apply to the discussion of infinite shifting.

Lemma 2.3. Let $A$ be a non-decreasing positive function on $[1,+\infty)$ and

$$
T(r)=\int_{1}^{r} \frac{A(t)}{t} \mathrm{~d} t, r \geq 1 .
$$

Set $\phi_{\varepsilon}(r)=\phi_{T, \varepsilon}(r)$ for a given $\varepsilon>0$. Then for all $1 \leq r<R \leq r+\phi_{\varepsilon}(r)^{\delta}$, $0<\delta<1$, we have

$$
T(R) \leq T(r)+4 e\left(\frac{1}{\phi_{\varepsilon}(r)}\right)^{1-\delta} T(r), r \notin E_{\varepsilon},
$$

where $\int_{E_{\varepsilon}} \frac{\mathrm{d} t}{t}<+\infty$ if $\phi_{\varepsilon}(r)>2^{1 /(1-\delta)}$ and $\phi_{\varepsilon}(r)<r$. If $R=r+h$ for a fixed positive real number $h$, then $\delta=0$ can be chosen in (2.8) and in addition, if $T(r)$ is of finite order $\rho$, then

$$
T(r+h) \leq T(r)+4 h K \frac{T(r)}{r}, r \notin E,
$$

where $E$ has the logarithmic density less than $\rho \frac{\log 2}{\log K}$.

Proof. From the definition of $T$, it follows that

$$
\begin{aligned}
T(R) & =\int_{1}^{R} \frac{A(t)}{t} \mathrm{~d} t=T(r)+\int_{r}^{R} \frac{A(t)}{t} \mathrm{~d} t \\
& \leq T(r)+A(R) \log \frac{R}{r} \\
& \leq T(r)+\frac{R-r}{r} \frac{1}{\log \alpha} \int_{R}^{\alpha R} \frac{A(t)}{t} \mathrm{~d} t \\
& \leq T(r)+\frac{\alpha}{\alpha-1} \frac{R-r}{r} T(\alpha R),
\end{aligned}
$$

with $\alpha>1$ satisfying $\alpha R=r+\phi(r)$. Here and henceforth, simply we write $\phi(r)$ for $\phi_{\varepsilon}(r)$. In terms of (2.7), we have

$$
T(\alpha R)=T(r+\phi(r)) \leq e T(r), r \notin E_{\varepsilon},
$$


where $E_{\varepsilon}$ has finite logarithmic measure. Thus

$$
\begin{aligned}
\frac{\alpha}{\alpha-1} \frac{R-r}{r} & =\frac{r+\phi(r)}{r+\phi(r)-R} \frac{R-r}{r} \\
& \leq \frac{r+\phi(r)}{\phi(r)-\phi(r)^{\delta}} \frac{\phi(r)^{\delta}}{r} \\
& =\frac{r+\phi(r)}{r\left(\phi(r)^{1-\delta}-1\right)} \\
& \leq 4\left(\frac{1}{\phi(r)}\right)^{1-\delta},
\end{aligned}
$$

if $\phi^{1-\delta}(r)>2$ and $\phi(r)<r$. Combining the above inequalities yield (2.8). Let us take $R=r+h$, specially. Then we have

$$
\begin{aligned}
\frac{\alpha}{\alpha-1} \frac{R-r}{r} T(\alpha R) & \leq \frac{r+\phi(r)}{\phi(r)-h} \frac{h}{r} e T(r) \\
& \leq \frac{2 e h}{\phi(r)-h} T(r) \\
& \leq \frac{4 e h}{\phi(r)} T(r),
\end{aligned}
$$

if $\phi(r)>2 h$ and $r \notin E_{\varepsilon}$. If we take $\alpha$ such that $\alpha R=2 r$ and $R=r+h$, then we have

$$
\frac{\alpha}{\alpha-1} \frac{R-r}{r} T(\alpha R)=\frac{2 h}{r-h} T(2 r)<4 h \frac{T(2 r)}{r} .
$$

Therefore, if the order $\rho$ of $T(r)$ is finite, then in view of a result in Hayman [14, we have

$$
T(2 r) \leq K T(r), r \notin W,
$$

where $W$ has $\overline{\log \operatorname{dens}} W \leq \rho \frac{\log 2}{\log K}$. This produces the desired result.

Now we are in position to prove Theorem 2.1.

Proof of Theorem 2.1. Set $\mu=\frac{1}{2 \pi}(\Delta u)^{-}$and $\mu_{c}=\frac{1}{2 \pi}\left(\Delta u_{c}\right)^{-}$. Since $B(0, t)+c \subset$ $B(0, t+|c|)$, we have

$$
n_{s}\left(t, u_{c}\right)=\mu_{c}(B(0, t))=\mu(B(0, t)+c) \leq \mu(B(0, t+|c|))=n_{s}(t+|c|, u),
$$

by noting that $\mu$ is a Borel measure. From the definition of $N_{s}\left(r, u_{c}\right)$ it follows that

$$
\begin{aligned}
N_{s}\left(r, u_{c}\right) & =\int_{1}^{r} \frac{n_{s}\left(t, u_{c}\right)}{t} \mathrm{~d} t+\int_{0}^{1} \frac{n_{s}\left(t, u_{c}\right)-n_{s}\left(0, u_{c}\right)}{t} \mathrm{~d} t \\
& \leq \int_{1}^{r} \frac{n_{s}(t+|c|, u)}{t} \mathrm{~d} t+\int_{0}^{1} \frac{n_{s}\left(t, u_{c}\right)-n_{s}\left(0, u_{c}\right)}{t} \mathrm{~d} t \\
& =\int_{1+|c|}^{r+|c|} \frac{n_{s}(t, u)}{t-|c|} \mathrm{d} t+\int_{0}^{1} \frac{n_{s}\left(t, u_{c}\right)-n_{s}\left(0, u_{c}\right)}{t} \mathrm{~d} t \\
& =N_{s}(r+|c|, u)+|c| \int_{1+|c|}^{r+|c|} \frac{n_{s}(t, u)}{t(t-|c|)} \mathrm{d} t-n_{s}(0, u) \log (1+|c|)
\end{aligned}
$$




$$
\begin{aligned}
& -\int_{0}^{1+|c|} \frac{n_{s}(t, u)-n_{s}(0, u)}{t} \mathrm{~d} t+\int_{0}^{1} \frac{n_{s}\left(t, u_{c}\right)-n_{s}\left(0, u_{c}\right)}{t} \mathrm{~d} t \\
= & N_{s}(r+|c|, u)+\frac{|c|}{r} N_{s}(r+|c|, u)+|c| \int_{1+|c|}^{r+|c|} \frac{N_{s}(t, u)}{(t-|c|)^{2}} \mathrm{~d} t+O(1),
\end{aligned}
$$

where the equality $\frac{1}{t-|c|}=\frac{1}{t}+\frac{|c|}{t(t-|c|)}$ was used and the last equality resulted from the formula of integration by parts.

Since $N_{s}(r, u)$ is logarithmically convex, $\frac{N_{s}(r, u)-N_{s}(1, u)}{\log r}$ is increasing for $r \geq 1$. Therefore, for $1+|c| \leq t \leq r+|c|$, we have

$$
\frac{N_{s}(t, u)-N_{s}(1, u)}{\log t} \leq \frac{N_{s}(r+|c|, u)-N_{s}(1, u)}{\log (r+|c|)} .
$$

This implies that

so that

$$
\frac{N_{s}(t, u)}{\log t} \leq \frac{N_{s}(1, u)}{\log t}+\frac{N_{s}(r+|c|, u)}{\log (r+|c|)}
$$

$$
\begin{aligned}
\int_{1+|c|}^{r+|c|} \frac{N_{s}(t, u)}{(t-|c|)^{2}} \mathrm{~d} t & \leq \frac{N_{s}(r+|c|, u)}{\log (r+|c|)} \int_{1+|c|}^{r+|c|} \frac{\log t}{(t-|c|)^{2}} \mathrm{~d} t+N_{s}(1, u) \\
& \leq \frac{\int_{1}^{r} \frac{\log (t+|c|)}{t^{2}} \mathrm{~d} t}{\log (r+|c|)} N_{s}(r+|c|, u)+N_{s}(1, u) .
\end{aligned}
$$

Noting that

$$
\begin{gathered}
\int_{1}^{\infty} \frac{\log (t+|c|)}{t^{2}} \mathrm{~d} t=-\left.\frac{\log (t+|c|)}{t}\right|_{1} ^{\infty}+\int_{1}^{\infty} \frac{\mathrm{d} t}{t(t+|c|)} \\
=\log (1+|c|)+\frac{1}{|c|} \log (1+|c|),
\end{gathered}
$$

from the above inequalities it follows that

$$
N_{s}\left(r, u_{c}\right) \leq\left(1+\frac{|c|}{r}+\frac{(1+|c|) \log (1+|c|)}{\log (r+|c|)}\right) N_{s}(r+|c|, u)+N_{s}(1, u) .
$$

From (2.2) it follows that $\phi(r)=\max _{1 \leq t \leq r}\left\{\frac{t}{\max \left\{1, \log N_{s}(t, u)\right\}}\right\} \rightarrow \infty(r \rightarrow \infty)$. In terms of Lemma 2.1, we have

$$
N_{s}\left(r, u_{c}\right) \leq\left(1+\varepsilon_{1}(r)\right) N_{s}(r, u), r \in F_{1},
$$

for a set $F_{1}$ of $r$ with upper density 1 and $\varepsilon_{1}(r) \rightarrow 0(r \rightarrow \infty)$. The same argument implies that

$$
N_{s}(r, u) \leq\left(1+\varepsilon_{2}(r)\right) N_{s}\left(r, u_{c}\right), r \in F_{2},
$$

for a set $F_{2}$ of $r$ with upper density 1 and $\varepsilon_{2}(r) \rightarrow 0(r \rightarrow \infty)$. Then

$$
N_{s}(r, u) \sim N_{s}\left(r, u_{c}\right) \text {, as } r \in F_{1} \cap F_{2} \rightarrow \infty \text {. }
$$

It is obvious that $F=F_{1} \cap F_{2}$ has upper density 1 and $E=[1,+\infty) \backslash F$ has zero lower density. The result (2) follows the same way as above.

In view of Lemma 2.3, we can deduce the result (3) along the same way as above, for from (2.3) it follows that $\phi_{N_{s}, \varepsilon}(r) \rightarrow \infty(r \rightarrow \infty)$. 
The proof of Theorem 2.1 is completed.

In order to obtain a finer estimate than (2.10), we should carefully discuss the estimate of the integral term in (2.9).

If $N_{s}(r, u)$ has finite order $\lambda$, then it follows that for $\varepsilon>0$ there exists a constant $K_{\varepsilon}>0$ such that $N_{s}(r, u)<K_{\varepsilon}(r-|c|)^{\lambda+\varepsilon}, r \geq 1+|c|$. For $\varepsilon>0$ with $\lambda-1+\varepsilon \neq 0$, we can estimate

$$
\begin{aligned}
\int_{1+|c|}^{r+|c|} \frac{N_{s}(t, u)}{(t-|c|)^{2}} \mathrm{~d} t & \leq K_{\varepsilon} \int_{1+|c|}^{r+|c|} \frac{(t-|c|)^{\lambda+\varepsilon}}{(t-|c|)^{2}} \mathrm{~d} t \\
& \leq \frac{K_{\varepsilon}}{|\lambda-1+\varepsilon|}\left(1+r^{\lambda-1+\varepsilon}\right)
\end{aligned}
$$

This together with (2.9) produces

$$
N_{s}\left(r, u_{c}\right) \leq N_{s}(r+|c|, u)+O\left(r^{\lambda-1+\varepsilon}\right)+O(1) .
$$

By noting that

$$
\begin{aligned}
& N_{s}(r+|c|, u)-N_{s}(r, u)=\int_{r}^{r+|c|} \frac{n_{s}(t, u)}{t} \mathrm{~d} t \\
& \leq n_{s}(r+|c|, u) \log \left(1+\frac{|c|}{r}\right)=O\left(r^{\lambda-1+\varepsilon}\right),
\end{aligned}
$$

we have

$$
N_{s}(r+|c|, u)=N_{s}(r, u)+O\left(r^{\lambda-1+\varepsilon}\right)+O(1) .
$$

The same arguments as above yield the above equality (2.11) for $\mathcal{T}_{s}(r, u)$ of subharmonic functions $u$ with $\lambda$ replaced by the order $\rho$, especially for $\mathcal{T}(r, f)$ of holomorphic curves $f$ including the algebroid functions and $T(r, f)$ of meromorphic functions $f$. The asymptotic identity (2.11) was obtained by Chiang and Feng [3]. Using the lemma of logarithmic differences as a bridge, that is,

$$
m\left(r, \frac{w_{c}}{w}\right)=O\left(r^{\rho-1+\varepsilon}\right)
$$

they imply that

$$
T\left(r, w_{c}\right)=T(r, w)+O\left(r^{\rho-1+\varepsilon}\right)+O(\log r) .
$$

Our methods in this paper are different from theirs. It is obvious that the above equalities make no sense for $r$ with $T(r, w)=o\left(r^{\rho-1+\varepsilon}\right)$. For example, if the lower order of $w$ is less than $\rho-1$, then there exists an unbounded set $F$ of $r$ such that $T(r, w)=o\left(r^{\rho-1+\varepsilon}\right), r \in F$.

In view of Lemma 1.1.2 in [26], if

$$
\liminf _{r \rightarrow \infty} \frac{N_{s}(d r, u)}{N_{s}(r, u)} \geq d
$$

for some $d>1$, then

$$
\int_{1+|c|}^{r+|c|} \frac{N_{s}(t, u)}{(t-|c|)^{2}} \mathrm{~d} t \leq K \frac{N_{s}(r+|c|, u)}{r}+O(1) .
$$


Thus (2.9) yields

$$
N_{s}\left(r, u_{c}\right) \leq\left(1+\frac{|c|(K+2)}{r}\right) N_{s}(r+|c|, u) .
$$

We also deal with the finite lower order. Assume that $N_{s}(r, u)$ has the lower order $\mu<+\infty$ and the order $\lambda>1$. Then in view of Theorem 1.1.3 in [26], for any fixed set $E$ of the finite logarithmic measure and $\max \{1, \mu\}<\rho \leq \lambda$, there exists a sequence of the Polyá peak $\left\{r_{n}\right\}$ of order $\rho$ outside $E$ such that

$$
\begin{gathered}
N_{s}\left(r_{n}+|c|, u\right) \leq\left(1+\varepsilon_{n}\right)\left(1+\frac{|c|}{r_{n}}\right)^{\rho} N_{s}\left(r_{n}, u\right), \\
\frac{N_{s}(t, u)}{t^{\rho-\varepsilon_{n}}} \leq K \frac{N_{s}\left(r_{n}, u\right)}{r_{n}^{\rho-\varepsilon_{n}}}
\end{gathered}
$$

for $1 \leq t \leq r_{n}+|c|$ and $\varepsilon_{n} \rightarrow 0(n \rightarrow \infty)$. Then we have for $r=r_{n}$

$$
\begin{aligned}
\int_{1}^{r} \frac{N_{s}(t+|c|, u)}{t^{2}} \mathrm{~d} t & \leq K \frac{N_{s}\left(r_{n}, u\right)}{r_{n}^{\rho-\varepsilon_{n}}} \int_{1}^{r} \frac{(t+|c|)^{\rho-\varepsilon_{n}}}{t^{2}} \mathrm{~d} t \\
& \leq K \frac{N_{s}\left(r_{n}, u\right)}{r_{n}^{\rho-\varepsilon_{n}}}(1+|c|)^{\rho} \int_{1}^{r} t^{\rho-2-\varepsilon_{n}} \mathrm{~d} t \\
& \leq K(1+|c|)^{\rho} \frac{N_{s}\left(r_{n}, u\right)}{r_{n}^{\rho-\varepsilon_{n}}} \frac{1}{\left|\rho-1-\varepsilon_{n}\right|}\left[1+r_{n}^{\rho-1-\varepsilon_{n}}\right] \\
& \leq \frac{2 K(1+|c|)^{\rho}}{\left|\rho-1-\varepsilon_{n}\right|} \frac{N_{s}\left(r_{n}, u\right)}{r_{n}}
\end{aligned}
$$

for all sufficiently large $n$ by noting that $r_{n}^{\rho-1-\varepsilon_{n}} \geq 1$.

For the case of the infinite order, in view of Lemma 1.1.3 in [26], there exist these $\left\{r_{n}\right\}$ such that the above inequality holds.

Throughout the above discussion, we assume that $c$ is a constant independent of $r$. Now we consider the case when $c$ is allowed to depend on $r$, as Chiang and Luo did in [4]. In view of Lemma 2.1, we can establish the following

Theorem 2.2. Let $u$ be a $\delta$-subharmonic function on $\mathbb{C}$ with (2.2). Assume that (2.12) holds. Then for all $c$ with $0<|c|<\phi^{\delta}(r)$ and $0<\delta<1$, we have

$$
N_{s}\left(r, u_{c}\right) \sim N_{s}(r, u) \text {, as } r \in E_{\delta} \rightarrow \infty,
$$

where $E_{\delta}$ is such that $\underline{\text { dens }} E_{\delta}=0$.

Proof. Under (2.12), we have (2.13). Applying Lemma 2.1 for $T=N_{s}$, we get

$$
N_{s}\left(r, u_{c}\right) \leq\left(1+\frac{|c|(K+2)}{r}\right)\left(1+4\left(\frac{1}{\phi(r)}\right)^{\frac{1-\delta}{2}}\right) N_{s}(r, u)\left(r \notin E_{\delta} \rightarrow \infty\right) \text {. }
$$

By noting that $n_{s}(t-|c|, u) \leq n\left(t, u_{c}\right)$ we can establish the opposite inequality. Therefore, we get (2.14).

We guess that (2.12) would not be necessary. But we have to look for another way to get (2.14) without (2.12). 


\section{Difference of a Logarithm}

Let $u$ be a $\delta$-subharmonic function on $\mathbb{C}$. Let $D$ be a domain on $\mathbb{C}$ surrounded by finitely many piecewise analytic curves. For any point $z \in D$ and a fixed complex number $c$ such that $z+c \in D$, we set $u_{c}(z)=u(z+c)$. Then, in view of the Poisson-Jensen formula (2.1), we have

$$
\begin{aligned}
u_{c}(z)-u(z)= & \frac{1}{2 \pi} \int_{\partial D} u(\zeta)\left(\frac{\partial G_{D}(z+c, \zeta)}{\partial \mathbf{n}}-\frac{\partial G_{D}(z, \zeta)}{\partial \mathbf{n}}\right) \mathrm{d} s \\
& +N_{s}(D, z+c, u)-N_{s}(D, z, u) \\
& +N_{s}(D, z,-u)-N_{s}(D, z+c,-u),
\end{aligned}
$$

so that, by noting that $G_{D}(z, w)>0$ for all $w \in D$ and using the fact that $(\Delta u)^{-}$ is a Borel measure, we have

$$
\begin{aligned}
\left(u_{c}(z)-u(z)\right)^{+} \leq & \frac{1}{2 \pi} \int_{\partial D}\left[u(\zeta)\left(\frac{\partial G_{D}(z+c, \zeta)}{\partial \mathbf{n}}-\frac{\partial G_{D}(z, \zeta)}{\partial \mathbf{n}}\right)\right]^{+} \mathrm{d} s \\
& +\left(N_{s}(D, z+c, u)-N_{s}(D, z, u)\right)^{+} \\
& +\left(N_{s}(D, z,-u)-N_{s}(D, z+c,-u)\right)^{+} \\
\leq & \frac{1}{2 \pi} \int_{\partial D} u^{+}(\zeta)\left(\frac{\partial G_{D}(z+c, \zeta)}{\partial \mathbf{n}}-\frac{\partial G_{D}(z, \zeta)}{\partial \mathbf{n}}\right)^{+} \mathrm{d} s \\
& +\frac{1}{2 \pi} \int_{\partial D}(-u)^{+}(\zeta)\left(\frac{\partial G_{D}(z, \zeta)}{\partial \mathbf{n}}-\frac{\partial G_{D}(z+c, \zeta)}{\partial \mathbf{n}}\right)^{+} \mathrm{d} s \\
& +N_{s}(D, z+c, u)+N_{s}(D, z,-u) .
\end{aligned}
$$

Let $B$ be a domain in $D$ surrounded by finitely many piecewise analytic curves. We want to estimate $m_{s}\left(B, a, u_{c}-u\right)$ for a fixed point $a \in B$. For a fixed point $w \in D$, in terms of the Poisson-Jensen formula (2.1), we have

$$
\begin{aligned}
m_{s}\left(B, a, G_{D}(z, w)\right) & =G_{D}(a, w)+\frac{1}{2 \pi} \int_{B} G_{B}(a, \zeta) \Delta_{\zeta} G_{D}(\zeta, w) \\
& =G_{D}(a, w)-\frac{1}{2 \pi} \int_{B} G_{B}(a, \zeta) \Delta_{\zeta} \log |\zeta-w| \\
& =G_{D}(a, w)-\int_{B} G_{B}(a, \zeta) \delta_{w} \\
& =G_{D}(a, w)-G_{B}(a, w) \chi_{B}(w),
\end{aligned}
$$

and

$$
\begin{aligned}
m_{s}\left(B, a, G_{D}(z+c, w)\right) & =G_{D}(a+c, w)-G_{B}(a, w-c) \chi_{B}(w-c) \\
& =G_{D}(a+c, w)-G_{B+c}(a+c, w) \chi_{B+c}(w),
\end{aligned}
$$

where $\Delta_{\zeta}=\Delta$ is the Laplacian with respect to $\zeta$, which is emphasized by writing $\zeta$ as a subscript, $\delta_{w}$ is the Dirac delta function at $w$ and $\chi_{B}$ is the characteristic function of $B$. For simplicity, define $N_{D, u}(z)=N_{s}(D, z, u)$ and $N_{D, u, c}(z)=$ $N_{s}(D, z+c, u)$. Then

$$
m_{s}\left(B, a, N_{D, u}\right)=\frac{1}{2 \pi} \int_{D} m_{s}\left(B, a, G_{D}(z, w)\right)(\Delta u)^{-}(w)
$$




$$
\begin{aligned}
& =\frac{1}{2 \pi} \int_{D}\left(G_{D}(a, w)-G_{B}(a, w) \chi_{B}(w)\right)(\Delta u)^{-}(w) \\
& =N_{s}(D, a, u)-N_{s}(B, a, u)
\end{aligned}
$$

and

$$
\begin{aligned}
m_{s}\left(B, a, N_{D, u, c}\right) & =\frac{1}{2 \pi} \int_{D} m_{s}\left(B, a, G_{D}(z+c, w)\right)(\Delta u)^{-}(w) \\
& =\frac{1}{2 \pi} \int_{D}\left(G_{D}(a+c, w)-G_{B+c}(a+c, w) \chi_{B+c}(w)\right)(\Delta u)^{-}(w) \\
& =N_{s}(D, a+c, u)-N_{s}(B+c, a+c, u) .
\end{aligned}
$$

Combining the above inequalities (3.1), (3.2) and (3.3) yields the following

Theorem 3.1. Let $u$ be a $\delta$-subharmonic function on $D$ and $B$ a domain such that $B \subset D$ and $B+c \subset D$. Then for any $a \in B$, we have

$$
\begin{aligned}
m_{s}\left(B, a, u_{c}-u\right) \leq & \frac{1}{2 \pi} \int_{\partial D}|u(\zeta)| m_{s}\left(B, a,\left|\frac{\partial G_{D}(z+c, \zeta)}{\partial \mathbf{n}}-\frac{\partial G_{D}(z, \zeta)}{\partial \mathbf{n}}\right|\right) \mathrm{d} s \\
& +N_{s}(D, a+c, u)-N_{s}(B+c, a+c, u) \\
& +N_{s}(D, a,-u)-N_{s}(B, a,-u) .
\end{aligned}
$$

Assume that $D$ is simply connected. Let $\phi_{a}: \mathbb{D} \rightarrow D$ be the Riemann mapping with $\phi_{a}(0)=a, \phi_{a}^{\prime}(0)>0$ and let $\psi_{a}$ be the inverse of $\phi_{a}$. Then

$$
\frac{\partial G_{D}(z, \zeta)}{\partial \mathbf{n}} \mathrm{d} s=-i \frac{\psi_{z}^{\prime}(\zeta)}{\psi_{z}(\zeta)} \mathrm{d} \zeta
$$

so that

$$
\left(\frac{\partial G_{D}(z+c, \zeta)}{\partial \mathbf{n}}-\frac{\partial G_{D}(z, \zeta)}{\partial \mathbf{n}}\right)^{+} \mathrm{d} s=\left(\frac{\psi_{z+c}^{\prime}(\zeta) \psi_{z}(\zeta)}{\psi_{z+c}(\zeta) \psi_{z}^{\prime}(\zeta)}-1\right)^{+} \frac{\partial G_{D}(z, \zeta)}{\partial \mathbf{n}} \mathrm{d} s
$$

where $\zeta \in \partial D$. Noting that $|b|=b^{+}+(-b)^{+}$for a complex number $b$, we have

$$
m_{s}\left(B, a,\left|\frac{\partial G_{D}(z+c, \zeta)}{\partial \mathbf{n}}-\frac{\partial G_{D}(z, \zeta)}{\partial \mathbf{n}}\right|\right)
$$$$
=m_{s}\left(B, a, \frac{\partial G_{D}(z+c, \zeta)}{\partial \mathbf{n}}-\frac{\partial G_{D}(z, \zeta)}{\partial \mathbf{n}}\right)+m_{s}\left(B, a, \frac{\partial G_{D}(z, \zeta)}{\partial \mathbf{n}}-\frac{\partial G_{D}(z+c, \zeta)}{\partial \mathbf{n}}\right) .
$$

From the definition of $N_{s}$, we have

$$
\begin{aligned}
N_{s}(D, c, u) & =\frac{1}{2 \pi} \int_{D} G_{D}(c, w)(\Delta u)^{-}(w) \\
& =\frac{1}{2 \pi} \int_{D} G_{D+c}(0, w-c)\left(\Delta u_{c}\right)^{-}(w-c) \\
& =\frac{1}{2 \pi} \int_{D+c} G_{D+c}(0, w)\left(\Delta u_{c}\right)^{-}(w) \\
& =N_{s}\left(D+c, 0, u_{c}\right) .
\end{aligned}
$$


Therefore, in view of the subordinate principle of the Green function, for simply connected domains $\hat{D}$ and $\tilde{D}$ such that $0 \in \tilde{D} \subset D+c \subset \hat{D}$, we have

$$
N_{s}\left(\tilde{D}, 0, u_{c}\right) \leq N_{s}(D, c, u) \leq N_{s}\left(\hat{D}, 0, u_{c}\right)
$$

These formulas apply to some special domains such as a disk or a sector. In this paper, we only establish Lemma of the logarithmic differences on a disk.

We discuss the case when $D=B(0, R)$ and $B=B(0, r)$ with $0<r<R$, where $B(0, r)$ denotes the disk centered at 0 with radius $r$. Then for $\zeta=R e^{i \theta}$ and $z=r e^{i \varphi}$, we have

$$
\begin{aligned}
& \left(\frac{\partial G_{D}(z+c, \zeta)}{\partial \mathbf{n}}-\frac{\partial G_{D}(z, \zeta)}{\partial \mathbf{n}}\right)^{+} \mathrm{d} s \\
= & \left(\frac{R^{2}-|z+c|^{2}}{|\zeta-z-c|^{2}}-\frac{R^{2}-|z|^{2}}{|\zeta-z|^{2}}\right)^{+} \mathrm{d} \theta \\
= & \frac{R^{2}-|z|^{2}}{|\zeta-z|^{2}}\left(\frac{R^{2}-|z+c|^{2}}{|\zeta-z-c|^{2}} \frac{|\zeta-z|^{2}}{R^{2}-|z|^{2}}-1\right)^{+} \mathrm{d} \theta \\
\leq & \frac{R^{2}-|z|^{2}}{|\zeta-z|^{2}}\left(\left(1+\frac{|z|^{2}-|z+c|^{2}}{R^{2}-|z|^{2}}\right) \frac{|\zeta-z|^{2}}{|\zeta-z-c|^{2}}-1\right)^{+} \mathrm{d} \theta \\
\leq & \frac{R^{2}-|z|^{2}}{|\zeta-z|^{2}}\left(\left(1+\frac{3|c||z|}{R^{2}-|z|^{2}}\right)\left(1+\frac{|c|}{|\zeta-z-c|}\right)^{2}-1\right) \mathrm{d} \theta \\
\leq & \left(\left(1+\frac{2|c|}{R-|z|}\right)\left(1+\frac{2}{|\zeta-z|}\right)^{2}-1\right) \frac{R^{2}-|z|^{2}}{|\zeta-z|^{2}} \mathrm{~d} \theta
\end{aligned}
$$

where we assume that $|\zeta-z| \geq \max \{1,2|c|\}$ and $|z|>|c|$. For simplicity, set $P=\frac{2|c|}{R-|z|}$ and $Q=\frac{1}{|\zeta-z|}$. Then the quantity in final bracket of the above inequality equals to

$$
\begin{aligned}
(1+P)(1+2 Q)^{2}-1 & =P+4 Q+4 Q^{2}+4 P Q+4 P Q^{2} \\
& <9 P+8 Q \leq \frac{18|c|+8}{R-|z|}
\end{aligned}
$$

Therefore, combining the above inequalities yields

$$
\left(\frac{\partial G_{D}(z+c, \zeta)}{\partial \mathbf{n}}-\frac{\partial G_{D}(z, \zeta)}{\partial \mathbf{n}}\right)^{+} \mathrm{d} s \leq \frac{18|c|+8}{R-|z|} \frac{R^{2}-|z|^{2}}{|\zeta-z|^{2}} \mathrm{~d} \theta .
$$

The same argument implies

$$
\left(\frac{\partial G_{D}(z, \zeta)}{\partial \mathbf{n}}-\frac{\partial G_{D}(z+c, \zeta)}{\partial \mathbf{n}}\right)^{+} \mathrm{d} s \leq \frac{18|c|+8}{R-|z|} \frac{R^{2}-|z|^{2}}{|\zeta-z|^{2}} \mathrm{~d} \theta .
$$

Thus it follows from (3.4) that

$$
m_{s}\left(B, 0,\left|\frac{\partial G_{D}(z+c, \zeta)}{\partial \mathbf{n}}-\frac{\partial G_{D}(z, \zeta)}{\partial \mathbf{n}}\right|\right) \mathrm{d} s \leq \frac{18(2|c|+1)}{R-|z|}
$$


by noting that

$$
\frac{1}{2 \pi} \int_{0}^{2 \pi} \frac{R^{2}-|z|^{2}}{|\zeta-z|^{2}} \mathrm{~d} \theta=\frac{1}{2 \pi} \int_{|\zeta|=R} \frac{\partial G_{D}(\zeta, z)}{\partial \mathbf{n}} \mathrm{d} s=1 .
$$

And from (3.5) it follows that

$$
N_{s}(D, c, u) \leq N_{s}\left(R+|c|, u_{c}\right) \text { and } N_{s}(B+c, c, u) \geq N_{s}\left(r-2|c|, u_{c}\right) .
$$

Now we can establish the lemma on the logarithmic differences for $\delta$-subharmonic functions.

Theorem 3.2. Let $u$ be a $\delta$-subharmonic function on $\mathbb{C}$. Then for any complex number $c$ and a real number $\delta$ with $0<\delta<1 / 2$, we have

$$
m_{s}\left(r, u_{c}-u\right)<436 e(1+|c|)\left(\frac{1}{\phi_{\varepsilon}(r)}\right)^{\delta} T_{s}(r, u), r \notin E_{\varepsilon},
$$

where $E_{\varepsilon}$ is a set in $r$ with finite logarithmic measure, for $1<\phi_{\varepsilon}(r)<r$ and $r>1+|c|$. If (2.4) holds for $T=T_{s}$, then the above inequality holds where $\phi_{\varepsilon}(r)$ is assumed to be $\phi(r)$ and $E_{\varepsilon}$ is the $E_{\delta}$ with $\underline{\text { dens }} E_{\delta}=0$.

Proof. In view of Theorem 3.1, (3.6) and (3.7), from the definition of $m_{s}(r, u)$ it follows that

$$
\begin{aligned}
m_{s}\left(r, u_{c}-u\right) \leq & \frac{18(2|c|+1)}{R-r}\left(m_{s}(R, u)+m_{s}(R,-u)\right) \\
& +\left(N_{s}\left(R+|c|, u_{c}\right)-N_{s}\left(r-2|c|, u_{c}\right)\right) \\
& +\left(N_{s}(R,-u)-N_{s}(r,-u)\right) .
\end{aligned}
$$

We want to find a real and positive function $V$ in $(0,+\infty)$ such that $V(r) \rightarrow$ $\infty(r \rightarrow \infty)$. To this end, solve the equation in $v$

$$
\begin{gathered}
\frac{R}{r-2|c|} \frac{v-r+3|c|}{R-v-|c|}=\frac{1}{V(r)}, v=\frac{(R-r+2|c|)(r-2|c|)}{R V(r)+r-2|c|}-3|c|+r, \\
\frac{1}{v-r}=\frac{R V(r)+r-2|c|}{(R-r-|c|)(r-2|c|)-3|c| R V(r)} \leq \frac{R(V(r)+1)}{(R-r-|c|)(r-2|c|)-3|c| R V(r)} .
\end{gathered}
$$

Taking $R=r+\hat{\phi}^{\delta}(r)$ and $V(r)+1=\hat{\phi}^{\delta / 2}(r)$ with $0<\delta<1,6|c| \leq \hat{\phi}^{\delta / 2}(r)$, $\hat{\phi}^{\delta}(r) \leq r$ and $\hat{\phi}(r) \rightarrow \infty(r \rightarrow \infty)$, we have $R \leq 2 r, v \geq 3|c|+r$ and $(R-r-$ $|c|)(r-2|c|) \geq 6|c| R V(r)$ so that

$$
\frac{1}{v-r} \leq \frac{2 R(V(r)+1)}{(R-r-|c|)(r-2|c|)} \leq 6\left(\frac{1}{\hat{\phi}(r)}\right)^{\delta / 2}, \frac{1}{V(r)} \leq 2\left(\frac{1}{\hat{\phi}(r)}\right)^{\delta / 2}
$$

Set $\mu=\frac{1}{2 \pi}(\Delta u)^{+}$and $n_{s}(t,-u)=\mu(B(0, t))$. Then

$$
\begin{aligned}
N_{s}(v,-u)-N_{s}(r,-u) & =\int_{r}^{v} \frac{n_{s}(t,-u)}{t} \mathrm{~d} t \leq n_{s}(v,-u) \log \frac{v}{r} \\
& \leq \frac{\log \frac{v}{r}}{\log \frac{R}{v}}\left(N_{s}(R,-u)-N_{s}(v,-u)\right)
\end{aligned}
$$




$$
\leq \frac{R}{r} \frac{v-r}{R-v} N_{s}(R,-u)
$$

The same calculation yields

$$
N_{s}\left(v+|c|, u_{c}\right)-N_{s}\left(r-2|c|, u_{c}\right) \leq \frac{R}{r-2|c|} \frac{v-r+3|c|}{R-v-|c|} N_{s}\left(R, u_{c}\right) .
$$

With $R$ replaced by $v$ in (3.8), in view of (2.10), it can be seen that

$$
\begin{aligned}
m_{s}\left(r, u_{c}-u\right) \leq & \frac{18(2|c|+1)}{v-r}\left(m_{s}(v, u)+m_{s}(v,-u)\right) \\
& +\left(N_{s}\left(v+|c|, u_{c}\right)-N_{s}\left(r-2|c|, u_{c}\right)\right) \\
& +\left(N_{s}(v,-u)-N_{s}(r,-u)\right) \\
\leq & \frac{36(2|c|+1)}{v-r} T_{s}(v, u)+\frac{1}{V(r)}\left(N_{s}\left(R, u_{c}\right)+N_{s}(R, u)\right) \\
\leq & \frac{36(2|c|+1)}{v-r} T_{s}(v, u)+\frac{2(1+|c|)}{V(r)} N_{s}(R, u)
\end{aligned}
$$

Inequality (3.9) together with the above inequalities yield

$$
m_{s}\left(r, u_{c}-u\right) \leq(436|c|+220)\left(\frac{1}{\hat{\phi}(r)}\right)^{\delta / 2} T_{s}\left(r+\hat{\phi}^{\delta}(r), u\right) .
$$

Take $\hat{\phi}(r)=\phi_{T, \varepsilon}(r)$ for $T(r)=T_{s}(r, u)$. Applying (2.7) to $T_{s}$ yields $T_{s}(R, u) \leq$ $T\left(r+\phi_{\varepsilon}(r), u\right) \leq e T_{s}(r, u), r \notin E_{\varepsilon}$ and then we obtain immediately the desired inequality.

We go to the final part of Theorem 3.2. If (2.4) holds, then taking $\hat{\phi}(r)=\phi(r) \rightarrow$ $\infty(r \rightarrow \infty)$, in view of Lemma 2.1, we have

$$
T_{s}\left(r+\phi^{\delta}(r), u\right) \leq e T_{s}(r, u), r \notin E_{\delta},
$$

with $\underline{\text { dens }} E_{\delta}=0$. We get the desired inequality.

It is clear that Theorem 1.2 and Theorem 1.3 are direct consequences of Theorem 3.2. We remark that $c$ is allowed to vary with $0 \leq|c| \leq o\left(\phi^{\delta}(r)\right)$ with $0<\delta<1 / 2$.

It is well-known that for a meromorphic function $f$ of finite order, we have

$$
m\left(r, \frac{f^{\prime}}{f}\right)=O(\log r)
$$

without any exceptional set of $r>1$. But the exceptional set is necessary for the case of logarithmic differences. Below let us find such an example of an entire function with finite order.

Example 3.1. Taking a sequence of positive numbers $\left\{r_{k}\right\}$ with $r_{k+1} \geq 2 r_{k}, r_{1}>6$ and a sequence of positive integers $\left\{n_{k}\right\}$, we consider the infinite product

$$
f(z)=f\left(z ; r_{k}, n_{k}\right)=\prod_{k=1}^{\infty}\left\{1-\left(\frac{z}{r_{k}}\right)^{n_{k}}\right\} .
$$


We will prove that for suitable $n_{k}, f$ is of finite order and

$$
m\left(r, \frac{f_{3}}{f}\right) \geq(1+o(1)) T\left(r, f_{3}\right) \text { and } T(r, f)=o\left(T\left(r, f_{3}\right)\right)
$$

as $r \in E \rightarrow \infty$, where $E$ has finite logarithmic measure and infinite linear measure.

Since $r_{k} \geq 2^{k}$, it follows that $f$ is an entire function on the complex plane. Let us estimate $N\left(r, \frac{1}{f}\right), N\left(r, \frac{1}{f_{3}}\right)$ and $T(r, f)$. We can write

$$
N\left(r, \frac{1}{f}\right)=\sum_{k=1}^{\infty} n_{k} \log ^{+} \frac{r}{r_{k}}
$$

and

$$
N\left(r, \frac{1}{f_{3}}\right)=\sum_{k=1}^{\infty} \sum_{j=0}^{n_{k}-1} \log ^{+} \frac{r}{\left|-3+\omega_{k}^{j} r_{k}\right|},
$$

where $\omega_{k}=e^{\frac{2 \pi}{n_{k}} i}$. Since

$$
\left|-3+r e^{i \theta}\right|^{2}=r^{2}-6 r \cos \theta+9 \leq r^{2}-3 \sqrt{2} r+9<(r-1)^{2},
$$

for $-\frac{\pi}{4} \leq \theta \leq \frac{\pi}{4}$ and $r>4$, we have

$$
N\left(r, \frac{1}{f_{3}}\right)>\sum_{k=1}^{\infty} \frac{1}{4} n_{k} \log ^{+} \frac{r}{r_{k}-1} .
$$

For $r_{s}-\frac{1}{2} \leq r \leq r_{s}$, we have

$$
N\left(r, \frac{1}{f}\right)=\sum_{k=1}^{s-1} n_{k} \log ^{+} \frac{r}{r_{k}}
$$

and

$$
\begin{aligned}
N\left(r, \frac{1}{f_{3}}\right) & >\frac{1}{4} \sum_{k=1}^{s} n_{k} \log ^{+} \frac{r}{r_{k}-1} \\
& =\frac{1}{4} \sum_{k=1}^{s-1} n_{k} \log ^{+} \frac{r}{r_{k}-1}+\frac{1}{4} n_{s} \log ^{+} \frac{r}{r_{s}-1} \\
& >\frac{1}{4} \sum_{k=1}^{s-1} n_{k} \log ^{+} \frac{r}{r_{k}-1}+\frac{n_{s}}{8 r_{s}} .
\end{aligned}
$$

Taking $n_{s}$ such that

$$
n_{s}>4 r_{s}\left(\log r_{s}\right)^{2} \sum_{k=1}^{s-1} n_{k}
$$

it can be seen that

$$
N\left(r, \frac{1}{f}\right)=o\left(N\left(r, \frac{1}{f_{3}}\right)\right), r_{s}-\frac{1}{2} \leq r \leq r_{s}
$$


as $s \rightarrow \infty$. Clearly, $E:=\bigcup_{s=1}^{\infty}\left[r_{s}-1 / 2, r_{s}\right]$ has positive, finite logarithmic measure and infinite linear measure. Since $f$ is entire, we have

$$
\begin{aligned}
T(r, f) & \leq \log M(r, f) \leq \sum_{k=1}^{\infty} \log \left\{1+\left(\frac{r}{r_{k}}\right)^{n_{k}}\right\} \\
& =\left(\sum_{r_{k}>r}+\sum_{r_{k} \leq r}\right) \log \left\{1+\left(\frac{r}{r_{k}}\right)^{n_{k}}\right\} \\
& \leq \sum_{r_{k}>r}\left(\frac{r}{r_{k}}\right)^{n_{k}}+\sum_{r_{k} \leq r}\left\{\log \left(\frac{r}{r_{k}}\right)^{n_{k}}+\log \left[1+\left(\frac{r_{k}}{r}\right)^{n_{k}}\right]\right\} \\
& \leq \sum_{k=0}^{\infty}\left(\frac{1}{2}\right)^{k n_{k}}+\sum_{r_{k} \leq r} n_{k} \log \frac{r}{r_{k}}+\sum_{r_{k} \leq r}\left(\frac{r_{k}}{r}\right)^{n_{k}} \\
& <2+N\left(r, \frac{1}{f}\right)+\sum_{r_{k} \leq r}\left(\frac{1}{2}\right)^{(k-1) n_{k}} \\
& \leq N\left(r, \frac{1}{f}\right)+4
\end{aligned}
$$

and so

$$
T(r, f)=o\left(N\left(r, \frac{1}{f_{3}}\right)\right)=o\left(T\left(r, f_{3}\right)\right), r_{s}-\frac{1}{2} \leq r \leq r_{s} .
$$

By noting that $m\left(r, f_{3}\right)=T\left(r, f_{3}\right)$ and $m(r, f)=T(r, f)$, we have

$$
m\left(r, \frac{f_{3}}{f}\right) \geq m\left(r, f_{3}\right)-m(r, f)=T\left(r, f_{3}\right)-T(r, f)=(1+o(1)) T\left(r, f_{3}\right)
$$

as $r \in E \rightarrow \infty$. It is easy to see that $f$ has finite order greater than or equal to 1 if $n_{s}$ is chosen to satisfy

$$
\limsup _{s \rightarrow \infty} \frac{\log n_{s}}{\log r_{s}}<\infty
$$

\section{Clunie Type Theorems}

Formulating a discrete analogue of the Painlevé property, that can be reliably applied to distinguish integrable discrete equations from the non-integrable ones, has been an important topic of study in the field of discrete integrable systems for over two decades. In the complex analytic setting it is natural to consider discrete equations as difference equations embedded into the complex plane, and to look at their meromorphic solutions [1]. It turns out that requiring the existence of admissible meromorphic solutions of slow growth is a property that can effectively single out Painlevé type equations for both discrete [9, 12] and delay differential equations [10]. We will consider first a general class of equations with as weak growth condition for the solutions as possible. 
A difference polynomial of a meromorphic function $w$ has the form

$$
P(z, \vec{w})=\sum_{\lambda \in I} a_{\lambda}(z) w(z)^{\lambda_{0}} w\left(z+c_{1}\right)^{\lambda_{1}} \ldots w\left(z+c_{n}\right)^{\lambda_{n}},
$$

where $\vec{w}(z)=\left(w(z), w\left(z+c_{1}\right), \ldots, w\left(z+c_{n}\right)\right), I=\left\{\left(\lambda_{0}, \lambda_{1}, \ldots, \lambda_{n}\right): \lambda_{j} \in \mathbb{N} \cup\{0\}\right\}$ is a finite multi-index set and all of $c_{j}(1 \leq j \leq n)$ are non-zero complex constants and every $a_{\lambda}(z)$ is a given meromorphic function. The total degree, denoted by $\operatorname{deg}_{\vec{w}}(P)$, of $P(z, \vec{w})$ in $\vec{w}$ is defined as

$$
\operatorname{deg}_{\vec{w}}(P):=\max \left\{\sum_{j=0}^{n} \lambda_{j}: \lambda=\left(\lambda_{0}, \lambda_{1}, \ldots, \lambda_{n}\right) \in I\right\}
$$

and the degree, denoted by $\operatorname{deg}_{c_{j}}(P)$, of $P(z, \vec{w})$ in $w\left(z+c_{j}\right)(1 \leq j \leq n)$ as

$$
\hat{\lambda}_{c_{j}}=\operatorname{deg}_{c_{j}}(P):=\max \left\{\lambda_{j}: \lambda=\left(\lambda_{0}, \lambda_{1}, \ldots, \lambda_{n}\right) \in I\right\}
$$

and

$$
\hat{\lambda}_{0}=\operatorname{deg}_{0}(P):=\max \left\{\lambda_{0}: \lambda=\left(\lambda_{0}, \lambda_{1}, \ldots, \lambda_{n}\right) \in I\right\} .
$$

Thus the weight of a difference polynomial $P(z, \vec{w})$ in $\vec{w}$ is

$$
\hat{\kappa}(P)=\sum_{j=1}^{n} \hat{\lambda}_{c_{j}}
$$

and we set

$$
\kappa(P):=\max \left\{\sum_{j=1}^{n} \lambda_{j}: \lambda=\left(\lambda_{0}, \lambda_{1}, \ldots, \lambda_{n}\right) \in I\right\} .
$$

Obviously, $\hat{\kappa}(P) \geq \kappa(P)$.

Theorem 4.1. If the difference equation

$$
P(z, \vec{w})=R(z, w),
$$

where $P(z, \vec{w})$ is a difference polynomial in $\vec{w}$ with $P\left(z, 0, x_{1}, \ldots, x_{n}\right) \neq 0$ and $R(z, w)$ is a rational function in $w$, has a non-rational meromorphic solution $w$ with (1.6), then

$$
\operatorname{deg}_{w}(R) \leq \sum_{j=0}^{n} \hat{\lambda}_{c_{j}}=\hat{k}(P)+\hat{\lambda}_{0} .
$$

Obtaining more precise results requires a careful singularity analysis near the poles of the solution. In what follows, we investigate the number of poles of meromorphic solutions of difference equations in terms of the Clunie type theorems. The following lemma may be known.

Lemma 4.1. Let $f$ be a meromorphic function. Assume that

$$
\begin{aligned}
& P(z, w)=a_{n}(z) w^{n}+a_{n-1}(z) w^{n-1}+\ldots+a_{1}(z) w+a_{0}(z) \\
& Q(z, w)=b_{m}(z) w^{m}+b_{m-1}(z) w^{m-1}+\ldots+b_{1}(z) w+b_{0}(z) \not \equiv 0
\end{aligned}
$$


with $n=\operatorname{deg}_{w}(P)$ and $m=\operatorname{deg}_{w}(Q)$, and meromorphic coefficients $a_{j}(z)(0 \leq j \leq$ $n)$ and $b_{i}(z)(0 \leq i \leq m)$, and that they do not have common factors. Set

$$
R(z, w)=\frac{P(z, w)}{Q(z, w)} .
$$

Then

$$
m(r, P(z, f)) \leq n m(r, f)+\sum_{j=0}^{n} m\left(r, a_{j}\right)+n \log 2
$$

and

$$
\begin{aligned}
(n-m) m(r, f) \leq & m(r, R(z, f))+(n-m) m\left(r, \frac{1}{a_{n}}\right) \\
& +(n-m) \sum_{j=0}^{n-1} m\left(r, a_{j}\right)+\sum_{i=0}^{m} m\left(r, b_{i}\right) \\
& +(2 n-2 m+1) \log 2+(n-m) \log n+\log (m+1) .
\end{aligned}
$$

Proof. Let us begin by proving (4.2). Set $P_{j}(z, f)=a_{n} f^{n-j}(z)+\ldots+a_{j+1} f(z)+$ $a_{j}, 0 \leq j \leq n$. For a given $r>0$, we have

$$
\begin{aligned}
m(r, P(z, f)) & \leq m\left(r, f P_{1}(z, f)\right)+m\left(r, a_{0}\right)+\log 2 \\
& \leq m\left(r, P_{1}(z, f)\right)+m(r, f)+m\left(r, a_{0}\right)+\log 2 \\
& \leq m\left(r, P_{2}(z, f)\right)+2 m(r, f)+m\left(r, a_{1}\right)+m\left(r, a_{0}\right)+2 \log 2 \\
& \leq n m(r, f)+\sum_{j=0}^{n} m\left(r, a_{j}\right)+n \log 2 .
\end{aligned}
$$

To prove (4.3), set

$$
E=\left\{\theta:\left|f\left(r e^{i \theta}\right)\right|>1+\frac{2}{\left|a_{n}\left(r e^{i \theta}\right)\right|} \sum_{j=0}^{n-1}\left|a_{j}\left(r e^{i \theta}\right)\right|\right\}
$$

and

$$
F=\left\{\theta: 1 \leq\left|f\left(r e^{i \theta}\right)\right| \leq 1+\frac{2}{\left|a_{n}\left(r e^{i \theta}\right)\right|} \sum_{j=0}^{n-1}\left|a_{j}\left(r e^{i \theta}\right)\right|\right\} .
$$

Here we require that for $\theta \in E \cup F, z=r e^{i \theta}$ is not a pole of $f$ and neither a pole of $a_{j}(0 \leq j \leq n)$, nor a zero of $a_{n}$. Then the calculation below is reasonable. For $\theta \in E$, it is easily seen that

$$
\begin{aligned}
\left|P\left(r e^{i \theta}, f\right)\right| & \geq\left|a_{n}\left(r e^{i \theta}\right)\right|\left|f\left(r e^{i \theta}\right)\right|^{n}-\left|f\left(r e^{i \theta}\right)\right|^{n-1} \sum_{j=0}^{n-1}\left|a_{j}\left(r e^{i \theta}\right)\right| \\
& \geq\left|f\left(r e^{i \theta}\right)\right|^{n}\left(\left|a_{n}\left(r e^{i \theta}\right)\right|-\frac{1}{\left|f\left(r e^{i \theta}\right)\right|} \sum_{j=0}^{n-1}\left|a_{j}\left(r e^{i \theta}\right)\right|\right) \\
& \geq \frac{1}{2}\left|a_{n}\left(r e^{i \theta}\right)\right|\left|f\left(r e^{i \theta}\right)\right|^{n},
\end{aligned}
$$


so that for $\theta \in E$,

$$
\left|f\left(r e^{i \theta}\right)\right|^{n-m} \leq 2 \cdot \frac{1}{\left|a_{n}\left(r e^{i \theta}\right)\right|}\left|R\left(r e^{i \theta}, f\right)\right| \sum_{i=0}^{m}\left|b_{i}\left(r e^{i \theta}\right)\right| .
$$

From the definition of $m(r, f)$ it follows that

$$
\begin{aligned}
& (n-m) m(r, f)=(n-m) \frac{1}{2 \pi} \int_{E \cup F} \log \left|f\left(r e^{i \theta}\right)\right| \mathrm{d} \theta \\
\leq & \frac{1}{2 \pi} \int_{E} \log \left|R\left(r e^{i \theta}, f\right)\right| \mathrm{d} \theta+\frac{1}{2 \pi} \int_{E} \log \left|\frac{1}{a_{n}\left(r e^{i \theta}\right)}\right| \mathrm{d} \theta+\log 2 \\
& +\frac{1}{2 \pi} \int_{E} \log \sum_{i=0}^{m}\left|b_{i}\left(r e^{i \theta}\right)\right| \mathrm{d} \theta+(n-m) \frac{1}{2 \pi} \int_{F} \log \left(1+\frac{2}{\left|a_{n}\left(r e^{i \theta}\right)\right|} \sum_{j=0}^{n-1}\left|a_{j}\left(r e^{i \theta}\right)\right|\right) \mathrm{d} \theta \\
\leq & m(r, R(z, f))+(n-m) m\left(r, \frac{1}{a_{n}}\right)+(n-m) \sum_{j=0}^{n-1} m\left(r, a_{j}\right)+\sum_{i=0}^{m} m\left(r, b_{i}\right) \\
& +(2 n-2 m+1) \log 2+(n-m) \log n+\log (m+1)
\end{aligned}
$$

outside a discrete set of $r>0$ where $r=|z|$ is such that either $f$ or $a_{j}(0 \leq j \leq n)$ has a pole, or $a_{n}$ has a zero, on a circle of radius $r$, centred at the origin. But since $m(r, f)$ is continuous, it follows that (4.3) holds for all $r>0$.

In general, it is basically impossible to estimate $m(r, R(z, f))$ in terms of $m(r, f)$, if $R(z, w)$ is not a polynomial in $w$.

Theorem 4.2. Let $f$ be a meromorphic function and

$$
\begin{aligned}
& P(z, \vec{f})=\sum_{\lambda \in I} a_{\lambda}(z) f(z)^{\lambda_{0}} f\left(z+c_{1}\right)^{\lambda_{1}} \ldots f\left(z+c_{n}\right)^{\lambda_{n}} \\
& Q(z, \vec{f})=\sum_{\lambda \in J} b_{\lambda}(z) f(z)^{\lambda_{0}} f\left(z+c_{1}\right)^{\lambda_{1}} \ldots f\left(z+c_{n}\right)^{\lambda_{n}},
\end{aligned}
$$

where $I \cup J=\left\{\left(\lambda_{0}, \lambda_{1}, \ldots, \lambda_{n}\right): \lambda_{j} \in \mathbb{N} \cup\{0\}\right\}$ is a finite multi-index set and all of $c_{j}(1 \leq j \leq n)$ are non-zero complex constants, and the $a_{\lambda}(z)$ and $b_{\lambda}(z)$ are meromorphic coefficients. Then

$$
\begin{aligned}
m(r, P(z, \vec{f})) \leq & \operatorname{deg}_{\vec{w}}(P) m(r, f)+\left(\sum_{\lambda \in I}|\lambda|\right) \sum_{j=1}^{n} m\left(r, \frac{f\left(z+c_{j}\right)}{f(z)}\right) \\
& +\sum_{\lambda \in I} m\left(r, a_{\lambda}\right)+\operatorname{deg}_{\vec{w}}(P) \log 2|I|,
\end{aligned}
$$

and if, in addition, $P(z, \vec{f})$ has just one term of maximal total degree, and $Q(z, \vec{f})$ and $P(z, \vec{f})$ do not have common factors, letting $R(z, \vec{f})=\frac{P(z, \vec{f})}{Q(z, \vec{f})}$, then

$$
\begin{aligned}
& \left(\operatorname{deg}_{\vec{w}}(P)-\operatorname{deg}_{\vec{w}}(Q)\right) m(r, f) \\
\leq & m(r, R(z, \vec{f}))+\operatorname{deg}_{\vec{w}}(P) \sum_{j=1}^{n} \hat{\lambda}_{j} m\left(r, \frac{f(z)}{f\left(z+c_{j}\right)}\right)
\end{aligned}
$$




$$
\begin{aligned}
& +\left(\operatorname{deg}_{\vec{w}}(P) \sum_{|\lambda|<m, \lambda \in I}|\lambda|+\sum_{\lambda \in J}|\lambda|\right) \sum_{j=1}^{n} m\left(r, \frac{f\left(z+c_{j}\right)}{f(z)}\right) \\
& +\operatorname{deg}_{\vec{w}}(P) m\left(r, \frac{1}{a_{\hat{\lambda}}}\right)+\operatorname{deg}_{\vec{w}}(P) \sum_{|\lambda|<m} m\left(r, a_{\lambda}\right)+\sum_{\lambda \in J} m\left(r, b_{\lambda}\right)+C,
\end{aligned}
$$

where $m=\operatorname{deg}_{\vec{w}}(P), \hat{\lambda}=\left(\hat{\lambda_{0}}, \hat{\lambda_{1}}, \ldots, \hat{\lambda_{n}}\right) \in I$ with $|\hat{\lambda}|=m$ and $C$ is a constant only depending on $\operatorname{deg}_{\vec{w}}(P), \operatorname{deg}_{\vec{w}}(Q)$ and $|I|,|J|$ which denote the numbers of elements of $I$ and $J$ in turn.

Proof. Set $m=\operatorname{deg}_{\vec{w}}(P)$ and

$$
c_{\lambda}(z)=\prod_{j=1}^{n}\left(\frac{f\left(z+c_{j}\right)}{f(z)}\right)^{\lambda_{j}}, \lambda=\left(\lambda_{0}, \lambda_{1}, \ldots, \lambda_{n}\right) \in I \cup J .
$$

Then we can rewrite $P(z, \vec{f})$ into

$$
P(z, \vec{f})=\sum_{\lambda \in I} a_{\lambda} c_{\lambda} f(z)^{|\lambda|}=\left(\sum_{|\lambda|=m} a_{\lambda} c_{\lambda}\right) f(z)^{m}+\sum_{|\lambda|<m} a_{\lambda} c_{\lambda} f(z)^{|\lambda|},
$$

where $|\lambda|=\sum_{j=0}^{n} \lambda_{j}$. In terms of Lemma 4.1, we have

$$
\begin{aligned}
m(r, P(z, \vec{f})) \leq & m m(r, f)+\sum_{k=0}^{m} m\left(r, \sum_{|\lambda|=k} a_{\lambda} c_{\lambda}\right)+\operatorname{deg}_{\vec{w}}(P) \log 2 \\
\leq & m m(r, f)+\sum_{k=0}^{m} \sum_{|\lambda|=k} m\left(r, a_{\lambda} c_{\lambda}\right)+\sum_{k=0}^{m} \log \sum_{|\lambda|=k} 1+\operatorname{deg}_{\vec{w}}(P) \log 2 \\
\leq & m m(r, f)+\sum_{\lambda \in I} m\left(r, c_{\lambda}\right)+\sum_{\lambda \in I} m\left(r, a_{\lambda}\right)+\operatorname{deg}_{\vec{w}}(P) \log 2|I| \\
\leq & m m(r, f)+\sum_{\lambda \in I} \sum_{j=1}^{n} \lambda_{j} m\left(r, \frac{f\left(z+c_{j}\right)}{f(z)}\right) \\
& +\sum_{\lambda \in I} m\left(r, a_{\lambda}\right)+\operatorname{deg}_{\vec{w}}(P) \log 2|I| \\
\leq & m m(r, f)+\left(\sum_{\lambda \in I}|\lambda|\right) \sum_{j=1}^{n} m\left(r, \frac{f\left(z+c_{j}\right)}{f(z)}\right) \\
& +\sum_{\lambda \in I} m\left(r, a_{\lambda}\right)+\operatorname{deg}_{\vec{w}}(P) \log 2|I| .
\end{aligned}
$$

This is (4.4). A similar argument together with (4.3) yields

$$
\begin{aligned}
m m(r, f) & \leq m(r, P(z, \vec{f}))+m m\left(r, \frac{1}{\sum_{|\lambda|=m} a_{\lambda} c_{\lambda}}\right)+m \sum_{|\lambda|<m} m\left(r, a_{\lambda} c_{\lambda}\right)+O(1) \\
& \leq m(r, P(z, \vec{f}))+m m\left(r, \frac{1}{a_{\hat{\lambda}}}\right)+m \sum_{j=1}^{n} \hat{\lambda}_{j} m\left(r, \frac{f(z)}{f\left(z+c_{j}\right)}\right)
\end{aligned}
$$




$$
+m \sum_{|\lambda|<m}|\lambda| \sum_{|\lambda|<m} m\left(r, \frac{f\left(z+c_{j}\right)}{f(z)}\right)+m \sum_{|\lambda|<m} m\left(r, a_{\lambda}\right)+O(1),
$$

where $\lambda \in I$ and $\hat{\lambda}=\left(\hat{\lambda}_{0}, \ldots, \hat{\lambda}_{n}\right) \in I$ with $|\hat{\lambda}|=m$. Since

$$
m(r, P(z, \vec{f}) \leq m(r, R(z, \vec{f}))+m(r, Q(z, \vec{f})),
$$

applying (4.4) to $m(r, Q(z, \vec{f})$ and in view of (4.7)), we deduce (4.5)).

Clunie's Theorem in [5] on an estimate of Nevanlinna proximity of a differential polynomial produced from a certain differential polynomial equation has proven valuable in the study of value distribution of meromorphic solutions of non-linear differential equations. It is natural to study the difference analogues of Clunie Theorem and their applications in difference equations. We consider the difference equation

$$
U(z, \vec{w}) P(z, \vec{w})=Q(z, \vec{w}),
$$

where $U(z, \vec{w}), P(z, \vec{w})$ and $Q(z, \vec{w})$ are three difference polynomials with meromorphic coefficients. Assume that (4.8) has an admissible meromorphic solution, i.e., the coefficients are small functions of it. We want to know the number of poles of the solution and hence we consider estimate of the proximity function of the solution from the equation (4.8) with the help of the analogue of the Clunie Theorem. The case when $U(z, \vec{w})=w^{n}$ and $\operatorname{deg}_{\vec{w}}(Q) \leq n$ was investigated in [11]. Moreover, in [17], the case when $U(z, \vec{w})$ contains just one term of maximal total degree in $w$ and its shifts with $\operatorname{deg}_{\vec{w}}(Q) \leq \operatorname{deg}_{\vec{w}}(U)$ was considered, and in [15] the case when $P(z, \vec{w})$ is homogeneous and $U(z, \vec{w})=U(z, w)$ and $Q(z, \vec{w})=Q(z, w)$ are polynomial in $w$, which covers all difference equations (1.1) - (1.4) listed in the introduction. In fact, the difference equations in [15] contain many known Painlevé difference equations.

Now we make a more careful discussion and establish a new difference Clunie type theorem. For a meromorphic function $f$, by $S(r, f)$ we denote a quantity such that $S(r, f)=o(T(r, f))$ as $r \rightarrow \infty$ outside of a set with finite logarithmic measure. For a polynomial $P(z, \vec{x})$ in $\vec{x}=\left(x_{0}, x_{1}, \ldots, x_{n}\right)$, by $\operatorname{ord}_{0}(P)$ we denote the multiplicity of zero of $P$ as a function of $x_{0}$ at $x_{0}=0$. If $\operatorname{ord}_{0}(P)=0$, then $P(z, \vec{x})$ contains at least one term without $x_{0}$. For $U, P$ and $Q$ in (4.8), we introduce the notations:

$$
\begin{aligned}
d_{\vec{w}} & =\max \left\{\operatorname{deg}_{\vec{w}}(Q), \operatorname{deg}_{\vec{w}}(P)+\operatorname{deg}_{\vec{w}}(U)\right\}-\min \left\{\operatorname{deg}_{\vec{w}}(P), \operatorname{ord}_{0}(Q)\right\} \\
D_{\vec{w}} & =d_{\vec{w}}-\operatorname{deg}_{\vec{w}}(P) \\
& =\max \left\{\operatorname{deg}_{\vec{w}}(Q)-\operatorname{deg}_{\vec{w}}(P), \operatorname{deg}_{\vec{w}}(U)\right\}-\min \left\{\operatorname{deg}_{\vec{w}}(P), \operatorname{ord}_{0}(Q)\right\} \\
\tau_{\vec{w}} & =d_{\vec{w}}-\hat{\kappa}(P) .
\end{aligned}
$$

In the following theorem we consider equation (4.8) in the case where $U(z, \vec{w})$ and $Q(z, \vec{w})$ are specialized as polynomials in $w$ with meromorphic coefficients.

Theorem 4.3. Let $P(z, \vec{w})$ be a homogeneous difference polynomial in $\vec{w}$ with $\operatorname{ord}_{0}(P)=0$ and $\hat{\lambda}_{0}<\operatorname{deg}_{\vec{w}}(P)$ and let $U(z, w)$ and $Q(z, w)$ be both polynomials 
in $w$ without any common factors. Assume that the difference equation (4.8) has a transcendental meromorphic solution $w$ such that, for some $\varepsilon>0$,

$$
\limsup _{r \rightarrow \infty} \frac{(\log r)^{1+\varepsilon} \log T(r, w)}{r}=0 .
$$

Then

$$
\hat{\kappa}(P) \geq \max \left\{\operatorname{deg}_{w}(Q)-\hat{\lambda}_{0}, \operatorname{deg}_{w}(U)-\min \left\{\hat{\lambda}_{0}, \operatorname{ord}_{0}(Q)\right\}\right\} .
$$

Furthermore, $\hat{\kappa}(P) \geq D_{\vec{w}}$. If $D_{\vec{w}}>0$, then we have

$$
\frac{D_{\vec{w}}}{\hat{\kappa}(P)} T(r, w) \leq N(r, w)+S(r, w)
$$

if $\tau_{\vec{w}}>0$, then we have

$$
\frac{\tau_{\vec{w}}}{\operatorname{deg}_{\vec{w}}(P)} T(r, w) \leq N\left(r, \frac{1}{w}\right)+S(r, w)
$$

if $\hat{\kappa}(P)=D_{\vec{w}}$, that is, $\operatorname{ord}_{0}(Q) \leq \hat{\lambda}_{0}$ and

$$
\hat{\kappa}(P)=\operatorname{deg}_{w}(U)-\operatorname{ord}_{0}(Q) \geq \operatorname{deg}_{w}(Q)-\hat{\lambda}_{0}
$$

then

$$
N(r, w)=T(r, w)+S(r, w), N\left(r, \frac{1}{w}\right)=T(r, w)+S(r, w) .
$$

Proof. In terms of Theorem 4.2 and Theorem 1.4, we have

$$
m\left(r, \frac{P(z, \vec{w})}{w^{\operatorname{deg}_{\vec{w}}(P)}}\right)=S(r, w) .
$$

It follows from (4.8) that, in terms of Valiron's Theorem, we have

$$
\begin{aligned}
T\left(r, \frac{P(z, \vec{w})}{w^{\operatorname{deg}_{\vec{w}}(P)}}\right) & =T\left(r, \frac{Q(z, w)}{w^{\operatorname{deg}_{\vec{w}}(P)} U(z, w)}\right) \\
& =d_{\vec{w}} T(r, w)+S(r, w) .
\end{aligned}
$$

Combining (4.14) and (4.15) yields

$$
N\left(r, \frac{P(z, \vec{w})}{w^{\operatorname{deg}_{\vec{w}}(P)}}\right)=d_{\vec{w}} T(r, w)+S(r, w) .
$$

On the other hand, by noting that $\kappa(P)=\operatorname{deg}_{\vec{w}}(P)$ for the homogeneous difference polynomial $P(z, \vec{w})$ with $\operatorname{ord}_{0}(P)=0$, we have

$$
\begin{aligned}
N\left(r, \frac{P(z, \vec{w})}{w^{\operatorname{deg}_{\vec{w}}(P)}}\right) & \leq N_{0}(r, P(z, \vec{w}))+N\left(r, \frac{1}{w^{\kappa(P)}}\right) \\
& \leq \hat{\kappa}(P) N(r+h, w)+S(r, w)+\kappa(P) N\left(r, \frac{1}{w}\right) \\
& \leq \hat{\kappa}(P) N(r, w)+\kappa(P) N\left(r, \frac{1}{w}\right)+S(r, w),
\end{aligned}
$$


where $N_{0}$ means that the poles of only $w$ are not counted and $h=\max \left\{\left|c_{j}\right|: 1 \leq\right.$ $j \leq n\}$. Therefore, we have

$$
\begin{aligned}
& \kappa(P) m\left(r, \frac{1}{w}\right)+\left(d_{\vec{w}}-\kappa(P)\right) T(r, w) \\
& \leq \hat{\kappa}(P) N(r, w)+S(r, w)
\end{aligned}
$$

and

$$
\begin{aligned}
& \hat{\kappa}(P) m(r, w)+\left(d_{\vec{w}}-\hat{\kappa}(P)\right) T(r, w) \\
& \leq \kappa(P) N\left(r, \frac{1}{w}\right)+S(r, w) .
\end{aligned}
$$

So we obtain

$$
\begin{aligned}
\hat{\kappa}(P) \geq & d_{\vec{w}}-\kappa(P) \\
= & \max \left\{\operatorname{deg}_{w}(Q)-\operatorname{deg}_{\vec{w}}(P), \operatorname{deg}_{w}(U)\right\} \\
& -\min \left\{\operatorname{deg}_{\vec{w}}(P), \operatorname{ord}_{0}(Q)\right\},
\end{aligned}
$$

by noting that $\operatorname{deg}_{\vec{w}}(P)=\kappa(P)$. In view of Theorem 4.1, we have

$$
\hat{\kappa}(P)+\hat{\lambda}_{0} \geq \max \left\{\operatorname{deg}_{w}(Q), \operatorname{deg}_{w}(U)\right\} .
$$

Now,

and

$$
\hat{\kappa}(P) \geq \operatorname{deg}_{w}(U)-\min \left\{\operatorname{deg}_{\vec{w}}(P), \operatorname{ord}_{0}(Q)\right\}
$$

so that

$$
\hat{\kappa}(P)+\hat{\lambda}_{0} \geq \operatorname{deg}_{w}(U),
$$

$$
\begin{aligned}
\hat{\kappa}(P) & \geq \operatorname{deg}_{w}(U)-\min \left\{\hat{\lambda}_{0}, \min \left\{\operatorname{deg}_{\vec{w}}(P), \operatorname{ord}_{0}(Q)\right\}\right\} \\
& =\operatorname{deg}_{w}(U)-\min \left\{\hat{\lambda}_{0}, \operatorname{deg}_{\vec{w}}(P), \operatorname{ord}_{0}(Q)\right\} \\
& =\operatorname{deg}_{w}(U)-\min \left\{\hat{\lambda}_{0}, \operatorname{ord}_{0}(Q)\right\},
\end{aligned}
$$

by noting that $\hat{\lambda}_{0} \leq \operatorname{deg}_{\vec{w}}(P)$. Also

$$
\hat{\kappa}(P) \geq \operatorname{deg}_{w}(Q)-\hat{\lambda}_{0} .
$$

These imply (4.9)).

Consider the case of $d_{\vec{w}}>\operatorname{deg}_{\vec{w}}(P)$, that is to say, $\operatorname{deg}_{w}(Q)>\operatorname{deg}_{\vec{w}}(P)+$ $\min \left\{\operatorname{deg}_{\vec{w}}(P), \operatorname{ord}_{0}(Q)\right\}$ or $\operatorname{deg}_{w}(U)>\min \left\{\operatorname{deg}_{\vec{w}}(P), \operatorname{ord}_{0}(Q)\right\}$. Then (4.10) follows from (4.18) and (4.11) from (4.19).

Assume that $\hat{\kappa}(P)=D_{\vec{w}}$. In view of (4.18), it can be seen that

$$
\operatorname{deg}_{\vec{w}}(P) m\left(r, \frac{1}{w}\right)+\hat{\kappa}(P) m(r, w)=S(r, w) .
$$

This immediately yields (4.13).

Let us make a careful discussion of the condition $\hat{\kappa}(P)=D_{\vec{w}}$. In terms of (4.9), we have

$$
\begin{aligned}
\hat{\kappa}(P) & =\max \left\{\operatorname{deg}_{w}(Q)-\hat{\lambda}_{0}, \operatorname{deg}_{w}(U)-\min \left\{\hat{\lambda}_{0}, \operatorname{ord}_{0}(Q)\right\}\right\} \\
& =\max \left\{\operatorname{deg}_{w}(Q)-\hat{\lambda}_{0}+\min \left\{\hat{\lambda}_{0}, \operatorname{ord}_{0}(Q)\right\}, \operatorname{deg}_{w}(U)\right\}-\min \left\{\hat{\lambda}_{0}, \operatorname{ord}_{0}(Q)\right\}
\end{aligned}
$$




$$
=\max \left\{\operatorname{deg}_{w}(Q)-\operatorname{deg}_{\vec{w}}(P), \operatorname{deg}_{w}(U)\right\}-\min \left\{\operatorname{deg}_{\vec{w}}(P), \operatorname{ord}_{0}(Q)\right\}
$$

so that

$$
\left.\min \left\{\hat{\lambda}_{0}, \operatorname{ord}_{0}(Q)\right\}\right\}=\min \left\{\operatorname{deg}_{\vec{w}}(P), \operatorname{ord}_{0}(Q)\right\}
$$

and

$$
\begin{gathered}
\max \left\{\operatorname{deg}_{w}(Q)-\hat{\lambda}_{0}+\min \left\{\hat{\lambda}_{0}, \operatorname{ord}_{0}(Q)\right\}, \operatorname{deg}_{w}(U)\right\} \\
=\max \left\{\operatorname{deg}_{w}(Q)-\operatorname{deg}_{\vec{w}}(P), \operatorname{deg}_{w}(U)\right\} .
\end{gathered}
$$

Since $\hat{\lambda}_{0}<\operatorname{deg}_{\vec{w}}(P)$, from (4.20) it follows that $\operatorname{ord}_{0}(Q) \leq \hat{\lambda}_{0}$. Thus (4.21) reduces to

$$
\begin{gathered}
\max \left\{\operatorname{deg}_{w}(Q)-\hat{\lambda}_{0}+\operatorname{ord}_{0}(Q), \operatorname{deg}_{w}(U)\right\} \\
=\max \left\{\operatorname{deg}_{w}(Q)-\operatorname{deg}_{\vec{w}}(P), \operatorname{deg}_{w}(U)\right\}
\end{gathered}
$$

Suppose that

$$
\operatorname{deg}_{w}(U) \leq \operatorname{deg}_{w}(Q)-\operatorname{deg}_{\vec{w}}(P) .
$$

Then by noting that $\operatorname{deg}_{w}(Q)-\hat{\lambda}_{0}+\operatorname{ord}_{0}(Q) \geq \operatorname{deg}_{w}(Q)-\operatorname{deg}_{\vec{w}}(P) \geq \operatorname{deg}_{w}(U)$, (4.22) yields $\operatorname{deg}_{w}(Q)-\hat{\lambda}_{0}+\operatorname{ord}_{0}(Q)=\operatorname{deg}_{w}(Q)-\operatorname{deg}_{\vec{w}}(P)$ and $\operatorname{so~} \operatorname{ord}_{0}(Q)=0$ and $\hat{\lambda}_{0}=\operatorname{deg}_{\vec{w}}(P)$, and a contradiction is derived. Therefore, $\operatorname{deg}_{w}(Q)-\operatorname{deg}_{\vec{w}}(P)<$ $\operatorname{deg}_{w}(U)$. It can be seen, by (4.22), that $\operatorname{deg}_{w}(U) \geq \operatorname{deg}_{w}(Q)-\hat{\lambda}_{0}+\operatorname{ord}_{0}(Q)$.

In conclusion, $\hat{\kappa}(P)=D_{\vec{w}}$ if and only if $\operatorname{ord}_{0}(Q) \leq \hat{\lambda}_{0}$ and

$$
\hat{\kappa}(P)=\operatorname{deg}_{w}(U)-\operatorname{ord}_{0}(Q) \geq \operatorname{deg}_{w}(Q)-\hat{\lambda}_{0} .
$$

These are (4.12).

We make a remark. If the condition of the growth for $w$ is (2.4), then the results in Theorem 4.3 also holds for $S(r, w)=o(T(r, w))$ outside a subset $E$ of $[1,+\infty)$ with $\underline{\text { dens }} E=0$.

Theorem 4.3 is an improvement of the results in [15], where the conditions for the existence of a meromorphic solution of the difference equation was not considered. The main results in [15] should be with $\hat{\kappa}(P)$ instead of $\kappa(P)$.

As an application of Theorem 4.3, we investigate the difference equation

$$
P(z, w, \bar{w}, \underline{w})=\bar{w} \underline{w}+\bar{w} w+w \underline{w}=\frac{Q(z, w)}{U(z, w)},
$$

where $Q(z, w)$ and $U(z, w)$ are both polynomials in $w$ and without any common factors. Then $\hat{\kappa}(P)=2, \hat{\lambda}_{0}=1, \operatorname{deg}_{\vec{w}}(P)=2$ so that $D_{\vec{w}}=\tau_{\vec{w}}$ and

$$
D_{\vec{w}}=\max \left\{\operatorname{deg}_{w}(Q)-2, \operatorname{deg}_{w}(U)\right\}-\min \left\{2, \operatorname{ord}_{0}(Q)\right\} \leq \hat{\kappa}(P)=2 .
$$

In view of Theorem 4.3, for $D_{\vec{w}}=\tau_{\vec{w}}=1$ we have

$$
\frac{1}{2} T(r, w) \leq N(r, w)+S(r, w) \text { and } \frac{1}{2} T(r, w) \leq N\left(r, \frac{1}{w}\right)+S(r, w) ;
$$

for $D_{\vec{w}}=2$, we have

$$
T(r, w) \leq N(r, w)+S(r, w) \text { and } T(r, w) \leq N\left(r, \frac{1}{w}\right)+S(r, w) .
$$


In view of (4.9) in Theorem 4.3, we have

$$
\max \left\{\operatorname{deg}_{w}(Q)-1, \operatorname{deg}_{w}(U)-\min \left\{1, \operatorname{ord}_{0}(Q)\right\}\right\} \leq \hat{\kappa}(P)=2 .
$$

Equivalently

$$
\operatorname{deg}_{w}(Q) \leq 3, \operatorname{deg}_{w}(U) \leq 2+\min \left\{1, \operatorname{ord}_{0}(Q)\right\} .
$$

Since $\operatorname{deg}_{w}(Q) \leq 3$, it follows that we only need to observe

$$
\operatorname{deg}_{w}(U)-2 \leq \min \left\{1, \operatorname{ord}_{0}(Q)\right\} .
$$

Let us divide into three cases to discuss.

(I) Assume that $\operatorname{ord}_{0}(Q)=0$. Then $\operatorname{deg}_{w}(U) \leq 2$ and

$$
D_{\vec{w}}=\max \left\{\operatorname{deg}_{w}(Q)-2, \operatorname{deg}_{w}(U)\right\}
$$

so that $0 \leq D_{\vec{w}} \leq 2$. When $D_{\vec{w}}=0$, we have $\operatorname{deg}_{w}(U)=0$ and $\operatorname{deg}_{w}(Q) \leq 2$. When $D_{\vec{w}}=1$, we have $\operatorname{deg}_{w}(U)=1, \operatorname{deg}_{w}(Q) \leq 3 \operatorname{or} \operatorname{deg}_{w}(U)=0, \operatorname{deg}_{w}(Q)=3$. When $D_{\vec{w}}=2(=\hat{\kappa}(P))$, we have $\operatorname{deg}_{w}(U)=2$ and $\operatorname{deg}_{w}(Q) \leq 3$. The corresponding difference equations have the following forms:

(4.24) $\bar{w} \underline{w}+\bar{w} w+w \underline{w}=a_{2} w^{2}+a_{1} w+a_{0}, a_{0} \neq 0, D_{\vec{w}}=0$;

$(4.25) \bar{w} \underline{w}+\bar{w} w+w \underline{w}=a_{3} w^{3}+a_{2} w^{2}+a_{1} w+a_{0}, a_{0} \neq 0, a_{3} \neq 0, D_{\vec{w}}=1$;

(4.26) $\bar{w} \underline{w}+\bar{w} w+w \underline{w}=\frac{a_{3} w^{3}+a_{2} w^{2}+a_{1} w+a_{0}}{w+b_{0}}, a_{0} \neq 0, D_{\vec{w}}=1$;

$(4.27) \bar{w} \underline{w}+\bar{w} w+w \underline{w}=\frac{a_{3} w^{3}+a_{2} w^{2}+a_{1} w+a_{0}}{w^{2}+b_{1} w+b_{0}}, a_{0} \neq 0, D_{\vec{w}}=2$.

(II) Assume that $\operatorname{ord}_{0}(Q)=1$. Then $\operatorname{deg}_{w}(U) \leq 3$ and

$$
D_{\vec{w}}=\max \left\{\operatorname{deg}_{w}(Q)-2, \operatorname{deg}_{w}(U)\right\}-1
$$

so that $-1 \leq D_{\vec{w}} \leq 2$. When $D_{\vec{w}}=0$, we have $\operatorname{deg}_{w}(U)=1, \operatorname{deg}_{w}(Q) \leq 3$ or $\operatorname{deg}_{w}(U)=0, \operatorname{deg}_{w}(Q)=3$. When $D_{\vec{w}}=1$, we have $\operatorname{deg}_{w}(U)=2$ and $\operatorname{deg}_{w}(Q) \leq$ 3. When $D_{\vec{w}}=2(=\hat{\kappa}(P))$, we have $\operatorname{deg}_{w}(U)=3$ and $\operatorname{deg}_{w}(Q) \leq 3$. When $D_{\vec{w}}=-1$, we have $\operatorname{deg}_{w}(U)=0$ and $1 \leq \operatorname{deg}_{w}(Q) \leq 2$. The corresponding difference equations have the following forms:

$$
\begin{aligned}
& \bar{w} \underline{w}+\bar{w} w+w \underline{w}=\frac{w\left(a_{2} w^{2}+a_{1} w+a_{0}\right)}{w+b_{0}}, a_{0} \neq 0, b_{0} \neq 0, D_{\vec{w}}=0 \\
& \bar{w} \underline{w}+\bar{w} w+w \underline{w}=w\left(a_{2} w^{2}+a_{1} w+a_{0}\right), a_{0} \neq 0, a_{2} \neq 0, D_{\vec{w}}=0 \\
& \bar{w} \underline{w}+\bar{w} w+w \underline{w}=\frac{w\left(a_{2} w^{2}+a_{1} w+a_{0}\right)}{w^{2}+b_{1} w+b_{0}}, a_{0} \neq 0, b_{0} \neq 0, D_{\vec{w}}=1 \\
& \bar{w} \underline{w}+\bar{w} w+w \underline{w}=\frac{w\left(a_{2} w^{2}+a_{1} w+a_{0}\right)}{w^{3}+b_{2} w^{2}+b_{1} w+b_{0}}, a_{0} \neq 0, b_{0} \neq 0, D_{\vec{w}}=2 ; \\
& \bar{w} \underline{w}+\bar{w} w+w \underline{w}=w\left(a_{1} w+a_{0}\right), a_{0} \neq 0, D_{\vec{w}}=-1 .
\end{aligned}
$$

(III) Assume that $2 \leq \operatorname{ord}_{0}(Q) \leq 3$ so that $2 \leq \operatorname{deg}_{w}(Q) \leq 3$. Then $\operatorname{deg}_{w}(U) \leq 3$ and

$$
D_{\vec{w}}=\max \left\{\operatorname{deg}_{w}(Q)-2, \operatorname{deg}_{w}(U)\right\}-2
$$


so that $-2 \leq D_{\vec{w}} \leq 1$. When $D_{\vec{w}}=0$, we have $\operatorname{deg}_{w}(U)=2$ and $2 \leq \operatorname{deg}_{w}(Q) \leq 3$. When $D_{\vec{w}}=1$, we have $\operatorname{deg}_{w}(U)=3$ and $2 \leq \operatorname{deg}_{w}(Q) \leq 3$. When $D_{\vec{w}}=-1$, we have $\operatorname{deg}_{w}(U)=1,2 \leq \operatorname{deg}_{w}(Q) \leq 3 \operatorname{or} \operatorname{deg}_{w}(U)=0, \operatorname{deg}_{w}(Q)=3$. When $D_{\vec{w}}=-2$, we have $\operatorname{deg}_{w}(U)=0$ and $\operatorname{deg}_{w}(Q)=2$. The corresponding difference equations have the following forms:

$$
\begin{aligned}
& \bar{w} \underline{w}+\bar{w} w+w \underline{w}=\frac{w^{2}\left(a_{1} w+a_{0}\right)}{w^{2}+b_{1} w+b_{0}}, b_{0} \neq 0, D_{\vec{w}}=0 ; \\
& \bar{w} \underline{w}+\bar{w} w+w \underline{w}=\frac{w^{2}\left(a_{1} w+a_{0}\right)}{w^{3}+b_{2} w^{2}+b_{1} w+b_{0}}, b_{0} \neq 0, D_{\vec{w}}=1 ; \\
& \bar{w} \underline{w}+\bar{w} w+w \underline{w}=w^{2}\left(a_{1} w+a_{0}\right), a_{1} \neq 0, D_{\vec{w}}=-1 ; \\
& \bar{w} \underline{w}+\bar{w} w+w \underline{w}=\frac{w^{2}\left(a_{1} w+a_{0}\right)}{w+b_{0}}, b_{0} \neq 0, D_{\vec{w}}=-1 ; \\
& \bar{w} \underline{w}+\bar{w} w+w \underline{w}=a_{2} w^{2}, a_{2} \neq 0, D_{\vec{w}}=-2 .
\end{aligned}
$$

Some of the 14 equations listed above can be ruled out according to the growth of the existing meromorphic solution.

Theorem 4.4. Let $w$ be a non-rational meromorphic solution of the difference equation

$$
\bar{w} \underline{w}+\bar{w} w+w \underline{w}=a_{3} w^{3}+a_{2} w^{2}+a_{1} w+a_{0}, a_{3} \neq 0 .
$$

Then for $a>1$ and $K>0$, we have

$$
T(r, w) \geq K D^{r}, r>1 .
$$

Proof. Suppose that $w$ has finitely many poles. For a sufficiently large $r$, we have

$$
\begin{aligned}
M(r, \bar{w} \underline{w}+\bar{w} w+w \underline{w}) & \leq M(r, \bar{w} \underline{w})+M(r, \bar{w} w)+M(r, w \underline{w}) \\
& \leq 3 M^{2}(r+1, w),
\end{aligned}
$$

where $M(r, *)$ denotes the maximal modulus of function $*$ on $|z|=r$. Also

$$
\begin{aligned}
& M\left(r, a_{3} w^{3}+a_{2} w^{2}+a_{1} w+a_{0}\right) \\
& \quad \geq M^{3}(r, w)\left(\left|a_{3}\left(z_{0}\right)\right|-\frac{1}{M(r, w)}\left(\left|a_{2}\left(z_{0}\right)\right|+\left|a_{1}\left(z_{0}\right)\right|+\left|a_{0}\left(z_{0}\right)\right|\right),\right.
\end{aligned}
$$

where $z_{0}$ with $\left|z_{0}\right|=r$ is such that $M(r, w)=\left|w\left(z_{0}\right)\right|$. So

$$
\frac{3}{2} \log M(r, w) \leq \log M(r+1, w)+S(r, w) .
$$

Now suppose that $w$ has infinitely many poles. When $z_{0}$ is a pole of $w$ with order $k$, we write $w\left(z_{0}\right)=\infty^{k_{0}}$. If $a_{j}\left(z_{0}\right) \neq 0, \infty(0 \leq j \leq 3)$, then

$$
(\bar{w} \underline{w}+\bar{w} w+w \underline{w})\left(z_{0}\right)=\infty^{3 k_{0}} .
$$

We obtain $\bar{w}\left(z_{0}\right)=\infty^{k_{1}}$ or $\underline{w}\left(z_{0}\right)=\infty^{k_{1}}$ with $k_{1} \geq \frac{3}{2} k_{0}$. Without loss of generality, we assume $w\left(z_{0}+1\right)=\infty^{k_{1}}$. If $a_{j}\left(z_{0}+1\right) \neq 0, \infty(0 \leq j \leq 3)$, then

$$
(\bar{w} \underline{w}+\bar{w} w+w \underline{w})\left(z_{0}+1\right)=\infty^{3 k_{1}} \text {. }
$$


It can now be seen that $w\left(z_{0}+2\right)=\infty^{k_{2}}$ with $k_{2} \geq \frac{3}{2} k_{1}$. Proceeding inductively, we have $w\left(z_{0}+n\right)=\infty^{k_{n}}$ with $k_{n} \geq\left(\frac{3}{2}\right)^{n} k_{0}$. Thus, we get

$$
\left(\frac{3}{2}\right)^{n} n(r, w) \leq n(r+n, w)+\sum_{j=0}^{3}\left(n\left(r+n, a_{j}\right)+n\left(r+n, \frac{1}{a_{j}}\right)\right) .
$$

so that we can deduce the inequality for $N(r, w)$, and therefore $N(r, w)>K\left(\frac{3}{2}\right)^{r}$ for some positive constant $K$.

In terms of Theorem 4.4, (4.25), (4.29) and (4.35) are ruled out of the above list if we assume the existence of admissible meromorphic solutions with the growth at most hyper-order 1 and minimal hyper-type.

Equations (4.31) and (4.34) can be rewritten into the following form

$$
(\bar{w}+w)(\underline{w}+w)=\frac{G(z, w)}{U(z, w)},
$$

where $\operatorname{deg}_{w}(G)=5, \operatorname{deg}_{w}(U)=3$. This derives a contradiction, see the paragraph after (1.4). Therefore, $\operatorname{deg}_{w}(U) \leq 2$ in the all equations listed above. Thus equations (4.31) and (4.34) are ruled out. It has been proven that the high density of poles of meromorphic solutions is a key in singling out the Painlevé type difference equations with the form (4.23). However, we emphasize that when $D_{\vec{w}} \leq 0$ from (4.10), we cannot always avoid the possibility that the solution $w$ has few poles. Lemma 2.5 in [25] shows that if $\operatorname{deg}_{w}(Q)=3$ in (4.23), then we have

$$
m(r, w)=T(r, w)+S(r, w) .
$$

Therefore, a meromorphic solution $w$ of (4.26) or (4.27) with $a_{3} \not \equiv 0,(4.28),(4.30)$ or (4.31) with $a_{2} \not \equiv 0,(4.33),(4.43)$ or (4.36) with $a_{1} \not \equiv 0$ satisfies (4.39). However, from (4.10), if $D_{\vec{w}}>0$, then (4.39) does not hold.

An observation to (4.27) yields that for $\operatorname{deg}_{w}(Q)<3$ and $\operatorname{deg}_{w}(U)=2$ in (4.23), (4.39) holds possibly.

Therefore, we have the following

Theorem 4.5. If the difference equation (4.23) have a meromorphic solution with the growth of (2.4), then (4.23) reduces to one of the following equations:

$$
\begin{aligned}
& \bar{w} \underline{w}+\bar{w} w+w \underline{w}=a_{2} w^{2}+a_{1} w+a_{0}, a_{0} \neq 0, D_{\vec{w}}=0 \\
& \bar{w} \underline{w}+\bar{w} w+w \underline{w}=\frac{a_{2} w^{2}+a_{1} w+a_{0}}{w+b_{0}}, a_{0} \neq 0, D_{\vec{w}}=1 \\
& \bar{w} \underline{w}+\bar{w} w+w \underline{w}=\frac{a_{2} w^{2}+a_{1} w+a_{0}}{w^{2}+b_{1} w+b_{0}}, a_{0} \neq 0, D_{\vec{w}}=2 \\
& \bar{w} \underline{w}+\bar{w} w+w \underline{w}=\frac{w\left(a_{2} w^{2}+a_{1} w+a_{0}\right)}{w+b_{0}}, a_{0} \neq 0, b_{0} \neq 0, D_{\vec{w}}=0 \\
& \bar{w} \underline{w}+\bar{w} w+w \underline{w}=\frac{w\left(a_{1} w+a_{0}\right)}{w^{2}+b_{1} w+b_{0}}, a_{0} \neq 0, b_{0} \neq 0, D_{\vec{w}}=1 \\
& \left.\bar{w} \underline{w}+\bar{w} w+w \underline{w}=a_{1} w+a_{0}\right), a_{0} \neq 0, D_{\vec{w}}=-1 ;
\end{aligned}
$$




$$
\begin{aligned}
& \bar{w} \underline{w}+\bar{w} w+w \underline{w}=\frac{w^{2}\left(a_{1} w+a_{0}\right)}{w^{2}+b_{1} w+b_{0}}, b_{0} \neq 0, D_{\vec{w}}=0 ; \\
& \bar{w} \underline{w}+\bar{w} w+w \underline{w}=\frac{w^{2}\left(a_{1} w+a_{0}\right)}{w+b_{0}}, b_{0} \neq 0, D_{\vec{w}}=-1 ; \\
& \bar{w} \underline{w}+\bar{w} w+w \underline{w}=a_{2} w^{2}, a_{2} \neq 0, D_{\vec{w}}=-2 .
\end{aligned}
$$

These above equations have been investigated in Zhang [24, [25] and Wen [22] to determine the conditions which their coefficients should satisfy.

Acknowledgements. We would like to express our gratitude to the referee for his valuable and careful comments in pointing out mistakes and improving this paper.

\section{REFERENCES}

[1] M. J. Ablowitz, R. G. Halburd and B. Herbst, On the extension of the Painlevé property to difference equations, Nonlinearity, 13(2000), 889-905.

[2] W. Cherry and Z. Ye, Nevanlinna's theory of value distribution, Springer-Verlag, Berlin, 2001.

[3] Y. M. Chiang and S. J. Feng, On the Nevanlinna characteristic of $f(z+\eta)$ and difference equations in the complex plane, Ramanujan J., 16(2008), 105-129.

[4] Y. M. Chiang and X. D. Luo, Difference Nevanlinna theories with vanishing and infinite periods, Michigan Math. J., 66(2017), 451-480.

[5] J. Clunie, On integral and meromorphic functions, J. London Math. Soc., 37(1962), 17-27.

[6] A. Edrei and W. H. J. Fuchs, Bounds for the number of deficient values of certain classes of meromorphic functions, Proc. London Math. Soc., 12(1962), 315-344.

[7] A. A. Goldberg and I. V. Ostrovskii, Value Distribution of Meromorphic Functions, Translations of Mathematical Monographs, vol. 236, 2008.

[8] B. Grammaticos, T. Tamizhmani, A. Ramani, K. M. Tamizhmani, Growth and integrability in discrete systems, J. Phys. A, Math. Gen., 34(2001), 3811-3821.

[9] R. G. Halburd and R. J. Korhonen, Finite-order meromorphic solutions and the discrete Painlevé equations, Proc. Lond. Math. Soc. (3), 94(2007), no. 2, 443-474.

[10] R. G. Halburd and R. J. Korhonen, Growth of meromorphic solutions of delay differential equations, Proc. Amer. Math. Soc., 145(2017), no. 6, 2513-2526.

[11] R. G. Halburd and R. Korhonen, Difference analogue of the lemma on the logarithmic derivative with applications to difference equations, J. Math. Anal. Appl., 314(2006), 477-487.

[12] R. Halburd, R. Korhonen and K. Tohge, Holomorphic curves with shift-invariant hyperplane preimages, Trans. Amer. Math. Soc., 366(2014), no. 8, 4267-4298.

[13] W. K. Hayman, Meromorphic Functions, Oxford Math. Monographs, Clarendon Press, Oxford, 1964.

[14] W. K. Hayman, On the characteristic of functions meromorphic in the plane and of their integrals, Proc. London Math. Soc. (3), 14A(1965), 93-128.

[15] R. Korhonen, A new Clunie type theorem for difference polynomials, J. Difference Equ. Appl., 17(2011), 387-400.

[16] R. Korhonen, N. Li and K. Tohge, Difference analogue of Cartain's second main thoerem for slowly moving periodic targets, Ann. Acad. Sci. Fenn. Math., 41(2016), 523-549.

[17] I. Laine and C. C. Yang, Clunie theorems for difference and $q$-difference polynomials, J. London Math. Soc., 76(2007), 556-566.

[18] A. Z. Mohon'ko, The Nevalinna characteristics of certain meromorphic functions, Teor. Funktsii Funktsional. Anal. i Prilozhen, 14 (1971), 83-87.

[19] T. Rainsford, Potential theory in the complex plane, Cambridge Univ. Press, 1995. 
[20] S. Shimomura, Entire solutions of a polynomial difference equations, J. Fac. Sci. Univ. Tokyo Sect. IA Math., 28(1981), 253-266.

[21] G. Valiron, Sur la dérivée des fonctions algébroídes, Bull. Soc. Math. France, 59(1931), 17-39.

[22] Z. T. Wen, Finite order solutions of meromorphic equations and difference Painlevé equations IV, Proc. Amer. Math. Soc., vol. 144, no. 10 (2016), 4247-4260.

[23] N. Yanagihara, Meromorphic solutions of some difference equations, Funkcialaj Ekvacioj, 23(1980), 309-326.

[24] J. L. Zhang, Meromorphic solutions of difference Painlevè IV Equations, Advances in Difference Equations, 2014, 2014:206.

[25] J. L. Zhang, Some results on difference Painlevè IV equations, J. Difference equations and applications, vol. 22, no.12 (2016), 1912-1929.

[26] J. H. Zheng, Value distribution of meromorphic functions, Springer-Verlag Berlin Heidelberg, 2010.

Department of Mathematical Sciences, Tsinghua University, P. R. China

E-mail address: zheng-jh@mail.tsinghua.edu.cn

Department of Physics and Mathematics, University of Eastern Finland, P. O. Box 111, 80101 JoensuU, Finland

E-mail address: risto.korhonen@uef.fi 